TITLE:

\title{
Bulk gravitational field and cosmological perturbations on the brane
}

AUTHOR(S):

Koyama, K; Soda, J

CITATION:

Koyama, K ...[et al]. Bulk gravitational field and cosmological

perturbations on the brane. PHYSICAL REVIEW D 2002, 65(2): 023514.

ISSUE DATE:

2002-01-15

URL:

http://hdl.handle.net/2433/49960

RIGHT:

Copyright 2002 American Physical Society 
PHYSICAL REVIEW D, VOLUME 65, 023514

\title{
Bulk gravitational field and cosmological perturbations on the brane
}

\author{
Kazuya Koyama \\ Graduate School of Human and Environment Studies, Kyoto University, Kyoto 606-8501, Japan
}

Jiro Soda

Department of Fundamental Sciences, FIHS, Kyoto University, Kyoto 606-8501, Japan

(Received 10 August 2001; published 26 December 2001)

\begin{abstract}
We investigate the effect of the bulk gravitational field on the cosmological perturbations on a brane embedded in 5D anti-de Sitter (AdS) spacetime. The effective 4D Einstein equations for the scalar cosmological perturbations on the brane are obtained by solving the perturbations in the bulk. Then the behavior of the corrections caused by the bulk gravitational field to the conventional 4D Einstein equation is determined. Two types of correction are found. First we investigate the corrections that become significant at scales below the AdS curvature scales and in the high energy universe with an energy density larger than the tension of the brane. The evolution equation for the perturbations on the brane is found and solved. Another type of correction is induced on the brane if we consider the bulk perturbations, which do not contribute to the metric perturbations but do contribute to matter perturbations. At low energy, they have an imaginary mass $m^{2}=$ $-(2 / 3) \mathbf{k}^{2}$ in the bulk where $\mathbf{k}$ is the $3 \mathrm{D}$ comoving wave number of the perturbations. They diverge at the horizon of the AdS spacetime. The induced density perturbations behave as sound waves with a sound velocity of $1 / \sqrt{3}$ in the low energy universe. At large scales, they are homogeneous perturbations that depend only on time and decay like radiation. They can be identified as the perturbations of dark radiation. They produce isocurvature perturbations in the matter dominated era. Their effects can be observed as shifts of the location and the height of the acoustic peak in the cosmic microwave background spectrum.
\end{abstract}

DOI: 10.1103/PhysRevD.65.023514

PACS number(s): 98.80.Cq, 04.50. $+\mathrm{h}$

\section{INTRODUCTION}

Recent developments in particle physics revive the old idea that we are living in a 4D brane in higher dimensional spacetime [1,2]. Since Randall and Sundrum proposed the fascinating model of the brane world, much work has been done concerning the consistency of the model with observations [3]. In their model, our three-brane universe is located in 5D anti-de Sitter (AdS) spacetime. The essence of the model is that the spacetime is effectively compactified with the curvature scale $l$ of the AdS spacetime. Thus, although gravity can propagate in the whole higher dimensional spacetime, 4D Newtonian gravity is reproduced at scales larger than $l$ on the brane.

After their work, the cosmological consequences of the model have been actively investigated [4-15]. The setup of the model is as follows. The action describing the brane world picture is given by

$$
\begin{aligned}
S= & \frac{1}{2 \kappa^{2}} \int d^{5} x \sqrt{-g}\left(\mathcal{R}^{5}+\frac{12}{l^{2}}\right)-\sigma \int d^{4} x \sqrt{-g_{\text {brane }}} \\
& +\int d^{4} x \sqrt{-g_{\text {brane }}} \mathcal{L}_{\text {matter }},
\end{aligned}
$$

where $\mathcal{R}^{5}$ is the 5D Ricci scalar, $l$ is the curvature radius of the AdS spacetime, and $\kappa^{2}=8 \pi G_{5}$, where $G_{5}$ is Newton's constant in the 5D spacetime. The brane has a tension $\sigma$ and the induced metric on the brane is denoted as $g_{\text {brane }}$. The tension $\sigma$ of the brane is taken as $\kappa^{2} \sigma=6 / l$ to ensure that the brane becomes Minkowski spacetime if there is no matter on the brane. Matter is confined to the 4D brane world and is described by the Lagrangian $\mathcal{L}_{\text {matter }}$. We will assume $Z_{2}$ symmetry across the brane. It has been shown that the spatially homogeneous and isotropic universe can also be embedded in this model. In order to study the consistency of the model with observations, it is necessary to study the behavior of the cosmological perturbations [16-18]. The cosmological perturbations in the brane world provide useful tests for the brane world idea. This is because the perturbations in the brane world interact with the bulk gravitational field; this is the inherent nature of perturbations in the brane world. Several formalisms and applications have been developed [19-29]. In particular, we showed that the evolution of the perturbations is the same as that obtained in conventional 4D theory at low energies when the Hubble horizon of the brane universe is larger than $l$. We also pointed out that the evolution of the perturbations changes significantly at high energy [19].

The purpose of this paper is to clarify the difference between the behavior of the perturbations in the brane world model and that in the conventional 4D model. For this purpose, it is desirable to obtain the effective 4D Einstein equations on the brane. There is some work that investigates the effective 4D Einstein equations with a projective approach [30,31]. The effective 4D Einstein equations are obtained as

$$
G_{\mu \nu}+\mathcal{E}_{\mu \nu}=\frac{\kappa^{2}}{l} T_{\mu \nu}+\kappa^{4} \Pi_{\mu \nu},
$$

where 


$$
\Pi_{\mu \nu}=-\frac{1}{4} T_{\mu \alpha} T_{\nu}^{\alpha}+\frac{1}{12} T_{\alpha}^{\alpha} T_{\mu \nu}+\frac{1}{24}\left(3 T_{\alpha \beta} T^{\alpha \beta}-\left(T_{\alpha}^{\alpha}\right)^{2}\right) g_{\mu \nu},
$$

and $\mathcal{E}_{\mu \nu}$ is the projected 5D Weyl tensor. In [29], the large scale cosmological perturbations are analyzed using Eq. (2). With a projective approach, the equations are used solely on the brane and the perturbations are not solved in the bulk. Although significant results can be obtained, this approach is clearly limited because the behavior of $\mathcal{E}_{\mu \nu}$ cannot be determined without solving the bulk perturbations. In a previous paper [19], we developed a method to solve the perturbations in the bulk. In this paper, using our method, we obtain the effective Einstein equation for the scalar cosmological perturbations by solving the perturbations in the bulk [see Eqs. (40) and (41) below]. Then we can determine the behavior of the corrections caused by the bulk gravitational field to the conventional 4D Einstein equations. This is an essential part of the work, predicting the cosmic microwave background (CMB) anisotropies in the brane world.

We will obtain the effective Einstein equations in two ways. First we derive the effective Einstein equations with a projective approach using the equations solely on the brane as in [29]. We observe the limitations of this approach. Then we construct the effective Einstein equations again by solving the bulk perturbations. The evolution of the perturbations on the brane is investigated using the effective Einstein equations. We concentrate our attention on the scalar perturbations on a brane in AdS spacetime. A new type of correction arises if we choose appropriate boundary conditions for the perturbations in the bulk so that they do not contribute to the metric perturbations but do contribute to the matter perturbations. They induce density perturbations on the brane, which behave as sound waves with sound velocity $1 / \sqrt{3}$ in the low energy universe. At large scales, they are homogeneous perturbations that depend only on time and decay like radiation. We will discuss the effects of these perturbations on the CMB spectrum.

The structure of the paper is as follows. In Sec. II, we construct the effective Einstein equation for the background spacetime in two ways as an example. In Sec. III, the effective Einstein equations for perturbations are constructed from the equations on the brane. Two types of correction to the conventional 4D Einstein equations are found. We see that a complete set of effective 4D Einstein equations cannot be derived from equations solely on the brane. In Sec. IV, the effective Einstein equations are obtained again by solving the perturbations in the bulk and imposing the junction conditions. A complete set of equations is obtained. We find again two types of correction, but now they are obtained according to the boundary conditions of the perturbations in the bulk. In Sec. V, we take the boundary condition that the perturbations do not diverge at the horizon of the AdS spacetime, and investigate the modifications of the evolution. In Sec. VI, we allow the existence of perturbations that do not contribute to the metric perturbations but do contribute to the matter perturbations. The modifications of the evolution caused by these perturbations are studied. In Sec. VII, we summarize the results. In Appendix A, the equations used in Sec. II are derived. In Appendix B, we review the formalism needed to solve the perturbations in the bulk and impose the junction conditions. Then the effective Einstein equations are obtained. In Appendix C, the generation of the primordial fluctuations is discussed. The Mukhanov equation for the inflaton confined to the brane is obtained.

\section{BACKGROUND SPACETIME}

It is instructive to consider the background spacetime as an example for constructing the effective Einstein equations. We take the background metric as

$$
d s^{2}=e^{2 \beta(y, t)}\left(d y^{2}-d t^{2}\right)+e^{2 \alpha(y, t)} \delta_{i j} d x^{i} d x^{j} .
$$

We will denote the power series expansion near the brane as

$$
\alpha(y, t)=\alpha_{0}(t)+\alpha_{1}(t)|y|+\frac{\alpha_{2}(t)}{2} y^{2}+\cdots .
$$

The tension $\sigma$ of the brane is taken as $\kappa^{2} \sigma=6 / l$ and the 5D energy-momentum tensor of the matter confined to the brane is

$$
T_{N}^{M}=\operatorname{diag}(0,-\rho, p, p, p) \delta(y) .
$$

The calculations that are necessary to obtain the equations used in the following discussion are performed in Appendix A.

We first employ a projective approach to obtain the effective Friedmann equation. We use the power series expansion of the 5D Einstein equation to obtain the equations solely on the brane. From the junction conditions, the first derivatives of the metric with respect to $y$ are written by means of the matter on the brane. Then we can obtain equations that contain only the variables on the brane from the 5D Einstein equations, which do not contain the second derivatives of the metric with respect to $y$. From these equations on the brane, we can construct the effective Einstein equations on the brane. The junction conditions are given by

$$
\begin{aligned}
& \alpha_{1}(t)=-\frac{1}{l}-\frac{\kappa^{2} \rho(t)}{6}, \\
& \beta_{1}(t)=-\frac{1}{l}+\frac{\kappa^{2} \rho(t)}{3}+\frac{\kappa^{2} p(t)}{2},
\end{aligned}
$$

where we take $e^{\beta_{0}(t)}=1$. The equations for $\alpha_{0}$ and $\rho$ can be obtained from the power series expansion of the 5D Einstein equation near the brane. The $y^{0}$ th order of the $(y, 0)$ and $(y, y)$ components is given by

$$
\begin{aligned}
\ddot{\alpha}_{0}+2 \dot{\alpha}_{0}^{2} & =\frac{\kappa^{2}}{2 l}\left(\frac{\rho}{3}-p\right)-\frac{\kappa^{4} \rho(\rho+3 p)}{36}, \\
\dot{\rho}+3 \dot{\alpha}_{0}(\rho+p) & =0 .
\end{aligned}
$$

At low energies $\rho / \sigma \sim \kappa^{-2} l \rho \ll 1$, the former is identical with the trace part of the conventional 4D Einstein equations with 


$$
8 \pi G_{4}=\kappa^{2} / l
$$

where $G_{4}$ is Newton's constant in the 4D spacetime. The latter is the usual energy-momentum conservation law for the matter. The integration of the first equation gives the effective Friedmann equation:

$$
\dot{\alpha}_{0}^{2}=\frac{\kappa^{2}}{3 l} \rho+\frac{\kappa^{4} \rho^{2}}{36}+e^{-4 \alpha_{0}} C_{0},
$$

where $C_{0}$ is the constant of integration. This is the $(t, t)$ component of the effective Einstein equations. In conventional 4D Einstein theory, the $(t, t)$ component of the Einstein equations gives

$$
\dot{\alpha}_{0}^{2}=\frac{8 \pi G_{4}}{3} \rho
$$

Thus the constant of integration $C_{0}$ should be zero in order to match the 4D Einstein theory at low energy. However, a nonzero $C_{0}$ is not forbidden in the brane world. Indeed, it is known that $C_{0}$ is related to the mass of the 5D AdSSchwartzschild black hole. Thus the nonzero $C_{0}$ indicates the effect of the bulk. The lesson is that even if we have a complete set of equations (8) for $\alpha_{0}$ and $\rho$ which are identical with those in conventional 4D theory at low energy, the correction to the Friedmann equation can exist. Because the term proportional to $C_{0}$ in Eq. (10) behaves like radiation, it is often called dark radiation. The important point is that we cannot determine $C_{0}$ from the equations on the brane (8). We need a different method to determine $C_{0}$ which describes the effect of the bulk.

Another way is to solve the 5D Einstein equation in the bulk. We should impose the boundary conditions (7) on the brane. The equations for $\beta$ and $\alpha$ in the bulk are given by

$$
\begin{aligned}
& -\ddot{\beta}+\beta^{\prime \prime}-\frac{1}{l^{2}} e^{2 \beta}=0, \\
& -\ddot{\alpha}+\alpha^{\prime \prime}-\frac{1}{l^{2}} e^{2 \beta}=0,
\end{aligned}
$$

where we assumed that the bulk is purely AdS spacetime without Schwartzschild mass (see Appendix A). We can obtain the solution

$$
e^{2 \beta(y, t)}=4 \frac{f^{\prime}(u) g^{\prime}(v)}{[f(u)-g(v)]^{2}}, \quad e^{2 \alpha(y, t)}=\frac{1}{[f(u)-g(v)]^{2}},
$$

where $u=(t-y) / l, v=(t+y) / l$, and $f(u)$ and $g(v)$ are arbitrary functions. Thus the matter on the brane is written using $f$ and $g$ from the junction conditions (7) as

$$
\alpha_{1}=\frac{1}{l}\left(\frac{f(t / l)^{\prime}+g(t / l)^{\prime}}{f(t / l)-g(t / l)}\right)=-\frac{1}{l}-\frac{\kappa^{2} \rho}{6} .
$$

Usually, we find the solutions of $f$ and $g$ from the junction condition (14). However, it is difficult to find solutions for the perturbations in this way. Thus we propose a new way to find the solutions, namely, transforming the junction condition (14) into the effective 4D Einstein equation. From Eq. (13), we obtain

$$
\begin{aligned}
\dot{\alpha}_{0} & =-\frac{1}{l}\left(\frac{f(t / l)^{\prime}-g(t / l)^{\prime}}{f(t / l)-g(t / l)}\right), \\
e^{2 \beta_{0}} & =4 \frac{f(t / l)^{\prime} g(t / l)^{\prime}}{[f(t / l)-g(t / l)]^{2}}=1 .
\end{aligned}
$$

Then the term written using $f$ and $g$ in Eq. (14) can be written using the metric $\alpha_{0}$. We find $\alpha_{1}^{2}=\dot{\alpha}_{0}^{2}+1 / l^{2}$. Thus the junction condition (14) gives the effective Friedmann equation on the brane:

$$
\dot{\alpha}_{0}^{2}=\frac{\kappa^{2}}{3 l} \rho+\frac{\kappa^{4} \rho^{2}}{36} .
$$

Comparing this with Eq. (10), we can determine $C_{0}=0$ for the AdS bulk. We will consider the perturbations on this background.

\section{EFFECTIVE EINSTEIN EQUATIONS FROM EQUATIONS ON THE BRANE}

In this section we will derive the effective 4D Einstein equations for scalar cosmological perturbations with a projective approach. The perturbed 5D energy-momentum tensor is taken as

$$
\delta T_{N}^{M}=\left(\begin{array}{ccc}
0 & 0 & 0 \\
0 & -\delta \rho & -(\rho+p) e^{\alpha_{0}} v_{, i} \\
0 & (\rho+p) e^{-\alpha_{0}} v_{, i} & \delta p \delta_{i j}
\end{array}\right) \delta(y)
$$

where we assume that the isotropic stress of the matter perturbations vanishes. The perturbed metric on the brane is taken as

$$
d s_{\text {brane }}=-\left(1+2 \Phi_{0}\right) d t^{2}+e^{2 \alpha_{0}(t)}\left(1-2 \Psi_{0}\right) \delta_{i j} d x^{i} d x^{j}
$$

As we did in the background case, we obtain the equations on the brane from a power series expansion of the 5D Einstein equations. From the $(y, y),(y, 0)$, and $(y, i)$ components of the 5D Einstein equations, we obtain [19]

$$
\begin{aligned}
& \ddot{\Psi}_{0}+4 \dot{\alpha}_{0} \dot{\Psi}_{0}+\dot{\alpha}_{0} \dot{\Phi}_{0}+2\left(\ddot{\alpha}_{0}+2 \dot{\alpha}_{0}^{2}\right) \Phi_{0}-\frac{1}{3} e^{-2 \alpha_{0}}\left(2 \nabla^{2} \Psi_{0}\right. \\
&\left.-\nabla^{2} \Phi_{0}\right)= \frac{\kappa^{2}}{3}\left(\frac{\beta_{1}}{2} \delta \rho-\frac{3 \alpha_{1}}{2} \delta p\right) \\
& \dot{\delta} \rho=(\rho+p)\left(3 \dot{\Psi}_{0}+e^{-\alpha_{0}} \nabla^{2} v\right) \\
&-3 \dot{\alpha}_{0}(\delta \rho+\delta p)
\end{aligned}
$$




$$
\left[(\rho+p) e^{\alpha_{0}} v\right]^{\cdot}=-3 \dot{\alpha}_{0}(\rho+p) e^{\alpha_{0}} v+\delta p+(\rho+p) \Phi_{0} .
$$

Equation (19) is the same as the trace of the conventional 4D Einstein equations at low energy and Eqs. (20) and (21) are the usual energy-momentum conservation law for the matter perturbations. From these equations, we construct the effective 4D Einstein equations. The Einstein equation gives a relation between the matter perturbations and the metric perturbations. Thus we try to write the matter perturbations in terms of the metric perturbations from Eqs. (19), (20), and (21). The equations can be regarded as differential equations for $\delta \rho, \delta p$, and $v$ with the source given by $\Phi_{0}$ and $\Psi_{0}$. Then the solutions for $\delta \rho, \delta p$, and $v$ are given by particular solutions written using $\Phi_{0}$ and $\Psi_{0}$ and the homogeneous solutions $\delta \rho_{\chi}, \delta p_{\chi}$, and $v_{\chi}$ which are independent of $\Phi_{0}$ and $\Psi_{0}$. The homogeneous solutions satisfy

$$
\begin{gathered}
\delta p_{\chi}=\frac{1}{3}\left(1+\frac{\ddot{\alpha}_{0}}{\alpha_{1}^{2}}\right) \delta \rho_{\chi}, \\
\dot{\delta} \rho_{\chi}=(\rho+p) e^{-\alpha_{0}} \nabla^{2} v_{\chi}-3 \dot{\alpha}_{0}\left(\delta \rho_{\chi}+\delta p_{\chi}\right), \\
\left((\rho+p) e^{\alpha_{0}} v_{\chi}\right)^{\cdot}=-3 \dot{\alpha}_{0}(\rho+p) e^{\alpha_{0}} v_{\chi}+\delta p_{\chi},
\end{gathered}
$$

where we used the background equations (A12). From these equations, we can construct the second order differential equation for $\delta \rho$. Putting

$$
\delta \rho_{\chi}=-\frac{1}{\alpha_{1} l} e^{-4 \alpha_{0}} \chi
$$

and substituting the first equation into the second and third equations, we get

$$
\begin{aligned}
\dot{\chi}= & \left(-\alpha_{1} l e^{2 \alpha_{0}}\right)(\rho+p) e^{\alpha_{0}} \nabla^{2} v_{\chi}, \\
\left((\rho+p) e^{\alpha_{0}} v_{\chi}\right)^{\cdot}= & -3 \dot{\alpha}_{0}(\rho+p) e^{\alpha_{0}} v_{\chi}-\frac{1}{\alpha_{1} l} e^{-4 \alpha_{0}} \frac{1}{3} \\
& \times\left(1+\frac{\ddot{\alpha}_{0}}{\alpha_{1}^{2}}\right) \chi .
\end{aligned}
$$

Then taking the time derivative of Eq. (24) and using Eq. (25), we obtain the equation for $\chi$ as

$$
\ddot{\chi}+\dot{\alpha}_{0}\left(1-\frac{\ddot{\alpha}_{0}}{\alpha_{1}^{2}}\right) \dot{\chi}-\frac{1}{3}\left(1+\frac{\ddot{\alpha}_{0}}{\alpha_{1}^{2}}\right) e^{-2 \alpha_{0}} \nabla^{2} \chi=0 .
$$

Particular solutions can be obtained perturbatively by assuming that $\left|e^{-2 \alpha_{0}} \nabla^{2} \Psi / \ddot{\Psi}_{0}\right| \ll 1$. The solutions up to order $\nabla^{4} \Psi_{0}$ including the homogeneous solutions using $\chi$ are given by

$$
\begin{aligned}
& -\frac{\kappa^{2} \alpha_{1}}{2} \delta \rho=-3\left(\dot{\alpha}_{0} \dot{\Psi}_{0}+\dot{\alpha}_{0}^{2} \Phi_{0}\right)+e^{-2 \alpha_{0}} \nabla^{2} \Psi_{0} \\
& -\frac{\kappa^{2} \alpha_{1}}{2} \delta \rho^{(4)}+\frac{\kappa^{2}}{2 l} \delta \rho_{\chi}, \\
& -\frac{\kappa^{2} \alpha_{1}}{2} \delta p=\ddot{\Psi}_{0}+\left(3 \dot{\alpha}_{0}-\frac{\dot{\alpha}_{0} \ddot{\alpha}_{0}}{\alpha_{1}^{2}}\right) \dot{\Psi}_{0}+\dot{\alpha}_{0} \dot{\Phi}_{0} \\
& +\left(2 \ddot{\alpha}_{0}-\frac{\dot{\alpha}_{0}^{2} \ddot{\alpha}_{0}}{\alpha_{1}^{2}}+3 \dot{\alpha}_{0}^{2}\right) \Phi_{0} \\
& +\frac{1}{3} e^{-2 \alpha_{0}} \nabla^{2} \Phi_{0}-\frac{1}{3}\left(1-\frac{\ddot{\alpha}_{0}}{\alpha_{1}^{2}}\right) \\
& \times e^{-2 \alpha_{0}} \nabla^{2} \Psi_{0}-\frac{\kappa^{2} \alpha_{1}}{2} \delta p^{(4)}+\frac{\kappa^{2}}{2 l} \delta p_{\chi}, \\
& -\frac{\kappa^{2} \alpha_{1}}{2}(\rho+p) e^{\alpha_{0}} v=\dot{\Psi}_{0}+\dot{\alpha}_{0} \Phi_{0}+\frac{1}{3} \alpha_{1} e^{-3 \alpha_{0}} \\
& \times \int d t^{\prime} e^{\alpha_{0}} \alpha_{1}^{-1} \\
& \times\left[\nabla^{2} \Phi_{0}-\left(1-\frac{\ddot{\alpha}_{0}}{\alpha_{1}^{2}}\right) \nabla^{2} \Psi_{0}\right] \\
& -\frac{\kappa^{2} \alpha_{1}}{2}(\rho+p) e^{\alpha_{0}} v^{(4)}+\frac{\kappa^{2}}{2 l}(\rho+p) \\
& \times e^{\alpha} v_{\chi},
\end{aligned}
$$

where $\delta \rho^{(4)}, \delta p^{(4)}$, and $v^{(4)}$ satisfy

$$
\begin{aligned}
& \dot{\delta} \rho^{(4)}-3 \dot{\alpha}_{0}\left(\delta \rho^{(4)}+\delta p^{(4)}\right)=-\frac{2}{3 \kappa^{2}} e^{-5 \alpha_{0}} \int d t^{\prime} e^{\alpha_{0}} \alpha_{1}^{-1} \\
& \times\left[\nabla^{4} \Phi_{0}-\left(1-\frac{\ddot{\alpha}_{0}}{\alpha_{1}^{2}}\right) \nabla^{4} \Psi_{0}\right], \\
& \delta p^{(4)}=\frac{1}{3}\left(1+\frac{\ddot{\alpha}_{0}}{\alpha_{1}^{2}}\right) \delta \rho^{(4)}, \\
& {\left[(\rho+p) e^{\alpha_{0}} v^{(4)}\right]^{\cdot}=-3 \dot{\alpha}_{0}(\rho+p) e^{\alpha_{0}} v^{(4)}+\delta p^{(4)},}
\end{aligned}
$$

and $\delta \rho_{\chi}, \delta p_{\chi}$, and $v_{\chi}$ are given by

$$
\begin{aligned}
\delta \rho_{\chi} & =e^{-4 \alpha_{0}} \chi\left(t, x^{i}\right), \\
\delta p_{\chi} & =\frac{1}{3}\left(1+\frac{\ddot{\alpha}_{0}}{\alpha_{1}^{2}}\right) e^{-4 \alpha_{0}} \chi\left(t, x^{i}\right), \\
(\rho+p) e^{\alpha_{0}} \nabla^{2} v_{\chi} & =e^{-2 \alpha_{0}} \dot{\chi}\left(t, x^{i}\right),
\end{aligned}
$$

where $\chi$ satisfies Eq. (26). 
Equations (27), (28), and (29) are the effective 4D Einstein equations in the brane world. The crucial difference between the background and the perturbations is that we cannot have a complete set of equations. In conventional 4D Einstein theory, in addition to these equations, we have the $(i \neq j)$ component of the Einstein equation

$$
\Psi_{0}-\Phi_{0}=0 .
$$

Then we have a closed set of equations. In the brane world, however, the corresponding equation derived from the projection of the 5D Einstein equation is

$$
\Psi_{0}-\Phi_{0}=e^{2 \alpha_{0}} E_{2}+N_{0},
$$

where $E$ is the $(i \neq j)$ component and $N$ is the $(y, y)$ component of the metric perturbations [see Eq. (B11) in Appendix $\mathrm{B}]$. The equation contains $E_{2}$, so it is not a closed system. The effect of the bulk perturbation $E_{2}$ and $N_{0}$ will alter the relation $\Phi_{0}=\Psi_{0}$. This is because the inhomogeneous fluctuations on the brane inevitably produce perturbations in the bulk, which give an effective anisotropic stress to the perturbations on the brane. We will see this in detail in Sec. IV. The important point is that we cannot know the behavior of the effective anisotropic stress caused by the bulk gravitational field, i.e., the right hand side of Eq. (31), with a projective approach.

To clarify the deviation from conventional 4D theory, we consider the perturbations at low energy with $\rho / \sigma \ll 1$ and then take

$$
\alpha_{1}^{2} \gg \dot{\alpha}_{0}^{2}, \quad \ddot{\alpha}_{0}
$$

If we take $\Phi_{0}=\Psi_{0}$, we have a complete set of equations with Eqs. (19), (20), and (21), which are identical with those obtained in conventional 4D theory. The interesting point is that, even if we take $\Psi_{0}=\Phi_{0}$, corrections to the matter perturbations can exist. Taking $\Psi_{0}=\Phi_{0}$, we find that $\delta \rho^{(4)}$, $\delta p^{(4)}$, and $v^{(4)}$ and the higher order solutions satisfy the homogeneous equations (22) which do not include $\Psi_{0}$ and $\Phi_{0}$. Thus they can be absorbed into $\delta \rho_{\chi}, \delta p_{\chi}$, and $v_{\chi}$. Then Eq. (27) becomes the same as the conventional $4 \mathrm{D}$ Einstein equation except for $\delta \rho_{\chi}, \delta p_{\chi}$, and $v_{\chi}$. Thus, even though we have a complete set of equations for metric perturbations and matter perturbations that are identical to those obtained in conventional 4D theory, corrections to the 4D effective Einstein equation can exist. We have already noted the similar situations in the background spacetime where the nonzero constant of integration $C_{0}$ gives a correction to the Friedmann equation. For the perturbations, $\chi$ plays the same role as $C_{0}$. At low energy, the equation for $\chi$ [Eq. (26)] becomes

$$
\chi^{\prime \prime}-\frac{1}{3} \nabla^{2} \chi=0,
$$

where a prime denotes the derivative with respect to the conformal time $\eta$. At large scales and at low energy, $\delta \rho_{\chi}$ is given by

$$
\delta \rho_{\chi}=C e^{-4 \alpha_{0}},
$$

where $\chi=C=$ const. Thus $\delta \rho_{\chi}$ can be regarded as the perturbations of the energy density of the dark radiation. At small scales they behave as sound waves with sound velocity $1 / \sqrt{3}$.

Hence we found two types of correction to the matter perturbations. One type of correction is given by the gradient of the metric perturbations that arise if the bulk gravitational field makes $\Phi_{0} \neq \Psi_{0}$. The other type of corrections $\chi$ is independent of the metric perturbations. At large scales they behave as dark radiation. Now we face the limitation of a projective approach, that is, the method using equations solely on the brane. We cannot obtain the relation between $\Phi_{0}$ and $\Psi_{0}$; thus the corrections given by the gradient of the metric perturbations cannot be determined. The existence of the correction given by $\chi$ also cannot be determined, as the constant of integration $C_{0}$ in the background cannot be determined in this approach. So far we have treated only equations that do not involve the second derivative with respect to $y$. As we showed in the background case, the evolution equation for the perturbations in the bulk should be solved in order to know the behavior of the corrections to the matter perturbations and the relation between $\Phi_{0}$ and $\Psi_{0}$.

\section{EFFECTIVE EINSTEIN EQUATIONS FROM BULK GRAVITATIONAL FIELD}

In this section, we solve the perturbations in the bulk and obtain the behavior of the corrections to the matter perturbations and the relation between $\Psi_{0}$ and $\Phi_{0}$. The formalism to solve the perturbations in the bulk was developed in [19]. In this section, we show only the results of the calculations. The detailed calculations are given in Appendix B. In the bulk, the perturbations satisfy the wave equation

$$
h^{\prime \prime}+3 \alpha^{\prime} h^{\prime}-\ddot{h}-3 \dot{\alpha} \dot{h}+e^{-2(\alpha-\beta)} \nabla^{2} h=0,
$$

where $h$ is the scalar perturbation in the bulk and we used the transverse-traceless gauge. It is difficult to solve this equation. The essence of our method is to a the coordinate transformation from Poincaré coordinates to Gaussian normal coordinates. The metric (4),(13) is obtained by coordinate transformation from the Poincare coordinates of the 5D AdS spacetime

$$
d s^{2}=\left(\frac{l}{z}\right)^{2}\left(d z^{2}-d \tau^{2}+\delta_{i j} d x^{i} d x^{j}\right)
$$

In these coordinates, the perturbations can be easily solved. Then the perturbations in the metric (4) can be obtained by performing the coordinate transformation:

$$
\begin{aligned}
& z=z(y, t)=l(f(u)-g(v))=l e^{-\alpha(y, t),} \\
& \tau=\tau(y, t)=l(f(u)+g(v)) .
\end{aligned}
$$

The solution of the perturbations $h$ can be written as 


$$
\begin{aligned}
h= & e^{-2 \alpha(y, t)} \int \frac{d^{3} \mathbf{k}}{(2 \pi)^{3}} \\
& \times \int d m E(m, \mathbf{k}) Z_{2}\left(m l e^{-\alpha(y, t)}\right) e^{-i \omega \tau(t, y)} e^{i \mathbf{k} \cdot \mathbf{x}},
\end{aligned}
$$

where $Z_{2}$ is defined as a combination of the Hankel functions of the first kind and of the second kind:

$$
Z_{2}(m z)=H_{2}^{(1)}(m z)+a(m) H_{2}^{(2)}(m z)
$$

and $\omega^{2}=m^{2}+\mathbf{k}^{2}$. So far $E(m, \mathbf{k})$ and $a(m)$ are arbitrary coefficients. We should impose the junction conditions for the perturbations (38) at the brane. As we showed for the background case, the junction conditions are nothing but the effective 4D Einstein equations. In a previous paper [19], we gave the matter perturbations in terms of $E(m, \mathbf{k})$ using the junction conditions. We also gave the metric perturbations in terms of $E(m, \mathbf{k})$. Thus we have the equations that correspond to Eqs. (14) and (15) in the case of the background spacetime. The effective Einstein equations can be obtained by combining these equations as is done in Eq. (16). The details can be found in Appendix B 2 and the results are given by

$$
\begin{aligned}
& -\frac{\kappa^{2} \alpha_{1}}{2} \delta \rho(\mathbf{k})=-3\left(\dot{\alpha}_{0} \dot{\Psi}_{0}+\dot{\alpha}_{0}^{2} \Phi_{0}\right)-e^{-2 \alpha_{0}} \mathbf{k}^{2} \Psi_{0} \\
& +\frac{1}{3} e^{-4 \alpha_{0}} \int d m E(m, \mathbf{k}) \mathbf{k}^{4} l^{2} Z_{0}\left(m l e^{-\alpha_{0}}\right) \\
& \times e^{-i \omega T(t)}, \\
& -\frac{\kappa^{2} \alpha_{1}}{2} \delta p(\mathbf{k})=\ddot{\Psi}_{0}+\left(3 \dot{\alpha}_{0}-\frac{\dot{\alpha}_{0} \ddot{\alpha}_{0}}{\alpha_{1}^{2}}\right) \dot{\Psi}_{0}+\dot{\alpha}_{0} \Phi_{0} \\
& +\left(2 \ddot{\alpha}_{0}-\frac{\dot{\alpha}_{0}^{2} \ddot{\alpha}_{0}}{\alpha_{1}^{2}}+3 \dot{\alpha}_{0}^{2}\right) \Phi_{0} \\
& -\frac{1}{3} e^{-2 \alpha_{0}} \mathbf{k}^{2} \Phi_{0} \\
& +\frac{1}{3}\left(1-\frac{\ddot{\alpha}_{0}}{\alpha_{1}^{2}}\right) e^{-2 \alpha_{0}} \mathbf{k}^{2} \Psi_{0} \\
& +\frac{1}{9}\left(1+\frac{\ddot{\alpha}_{0}}{\alpha_{1}^{2}}\right) e^{-4 \alpha_{0}} \\
& \times \int d m E(m, \mathbf{k}) \mathbf{k}^{4} l^{2} Z_{0}\left(m l e^{-\alpha_{0}}\right) \\
& \times e^{-i \omega T(t)},
\end{aligned}
$$

$$
\begin{aligned}
-\frac{\kappa^{2} \alpha_{1}}{2}(\rho+p) e^{\alpha_{0}} v(\mathbf{k})= & \dot{\Psi}_{0}+\dot{\alpha}_{0} \Phi_{0} \\
& +\frac{1}{3} e^{-3 \alpha_{0} \int d m E(m, \mathbf{k})} \\
& \times\left[\alpha_{1} i \omega \mathbf{k}^{2} l^{3} Z_{0}\left(m l e^{-\alpha_{0}}\right)\right. \\
& \left.-\dot{\alpha}_{0} m \mathbf{k}^{2} l^{3} Z_{1}\left(m l e^{-\alpha_{0}}\right)\right] e^{-i \omega T(t)},
\end{aligned}
$$

where we considered the Fourier components of the perturbations with respect to $x^{i}$ and denoted $\tau(0, t)=T(t)$. We can also obtain the metric perturbations in terms of $E(m, \mathbf{k})$ as

$$
\begin{aligned}
\Psi_{0}(\mathbf{k})= & \int d m E(m, \mathbf{k})\left(m l e^{-\alpha_{0}} Z_{1}\left(m l e^{-\alpha_{0}}\right)\right. \\
& \left.+\frac{1}{3}\left(\mathbf{k} l e^{-\alpha_{0}}\right)^{2} Z_{0}\left(m l e^{-\alpha_{0}}\right)\right) e^{-i \omega T(t)} \\
\Phi_{0}(\mathbf{k})= & \int d m E(m, \mathbf{k})\left(m l e^{-\alpha_{0}} Z_{1}\left(m l e^{-\alpha_{0}}\right)\right. \\
& \left.-\frac{1}{3}\left(\mathbf{k}^{2}+3 m^{2}\right) l^{2} e^{-2 \alpha_{0}} Z_{0}\left(m l e^{-\alpha_{0}}\right)\right) e^{-i \omega T(t)} \\
& +\left(\dot{\alpha}_{0} l\right)^{2} \int d m E(m, \mathbf{k})\left[m l e^{-\alpha_{0}} Z_{1}\left(m l e^{-\alpha_{0}}\right)\right. \\
& \left.-\left(\mathbf{k}^{2}+2 m^{2}\right) l^{2} e^{-2 \alpha_{0}} Z_{0}\left(m l e^{-\alpha_{0}}\right)\right] e^{-i \omega T(t)} \\
& -2 \alpha_{1} \dot{\alpha}_{0} l^{2} \int d m E(m, \mathbf{k}) \\
& \times\left(i \omega m l^{2} e^{-2 \alpha_{0}}\right) Z_{1}\left(m l e^{-\alpha_{0}}\right) e^{-i \omega T(t)}
\end{aligned}
$$

Equation (40) should be compared with Eqs. (27), (28), and (29). First let us identify the corrections (29), $\delta \rho_{\chi}, \delta p_{\chi}$, and $v_{\chi}$. There are two arbitrary coefficients $E(m, \mathbf{k})$ and $a(m)$ in the bulk perturbations (38). They should be determined by the boundary conditions in the bulk. The corrections (29) are independent of the metric perturbations. Thus, the perturbations in the bulk should not contribute to the metric perturbations but only to the matter perturbations. Then we impose the boundary condition that the metric perturbations vanish on the brane, i.e., $\Phi_{0}=\Psi_{0}=0$. From these two boundary conditions, the coefficients $E(m, \mathbf{k})$ and $a(m)$ are determined. Let us consider the low energy universe with $\dot{\alpha}_{0} l \ll 1$ and construct these perturbations explicitly. We first impose the boundary condition $\Psi_{0}=\Phi_{0}$. It can be implemented by choosing $E(m, \mathbf{k})$ to have a peak at

$$
2 \mathbf{k}^{2}+3 m^{2}=0
$$

Then the metric perturbations can be written as

$$
\Psi_{0}=\Phi_{0}=\frac{1}{2} E^{(\chi)}(\mathbf{k})\left(m_{k} l e^{-\alpha_{0}}\right)^{2} Z_{2}\left(m_{k} l e^{-\alpha_{0}}\right) e^{i \mathbf{k} \eta / \sqrt{3}},
$$


where we used $T=-\eta$ and $Z_{2}(z)=(2 / z) Z_{1}(z)-Z_{0}(z)$. It should be noted that $\eta$ is the conformal time [see Eq. (B7) in Appendix B]. We denoted $m_{k}=\sqrt{2 / 3} \mathbf{k} i$ and

$$
E^{(\chi)}(\mathbf{k})=E\left(m_{k}, \mathbf{k}\right) .
$$

Next, we impose the boundary condition that the metric perturbations vanish on the brane by choosing an appropriate coefficient $a(m)$. At low energy, we can neglect the time dependence in $m_{k} l e^{-\alpha_{0}}$ since

$$
\frac{(d / d t)\left(m_{k} l e^{-\alpha_{0}}\right)}{(d / d t)\left(e^{i \omega \eta}\right)} \sim \dot{\alpha}_{0} l \ll 1,
$$

where we used $d \eta / d t=e^{-\alpha_{0}}$. The condition $\Phi_{0}=\Psi_{0}=0$ can be implemented by taking $a(m)=a^{(\chi)}(m)$ where

$$
a^{(\chi)}(m)=-\frac{H_{2}^{(1)}\left(m_{k} l e^{-\alpha_{0}}\right)}{H_{2}^{(2)}\left(m_{k} l e^{-\alpha_{0}}\right)} .
$$

The important point is that these perturbations do contribute to the matter perturbations. The density perturbations $\delta \rho$ induced by these perturbations are given by

$$
\frac{\kappa^{2}}{2 l} \delta \rho=\frac{1}{3} e^{-4 \alpha_{0}} \mathbf{k}^{4} l^{2} E^{(\chi)}(\mathbf{k}) Z_{0}^{(\chi)}\left(m_{k} l e^{-\alpha_{0}}\right) e^{i \mathbf{k} \eta / \sqrt{3}},
$$

where $Z_{0}^{(\chi)}=H_{0}^{(1)}+a^{(\chi)}(m) H_{0}^{(2)}$. Because these perturbations do not contribute to the metric perturbations, they should be identified with $\delta \rho_{\chi}$. Indeed, from Eqs. (23) and (33), $\delta \rho_{\chi}$ is given by

$$
\delta \rho_{\chi}=e^{-4 \alpha_{0}} \chi, \quad \chi^{\prime \prime}+\frac{1}{3} \mathbf{k}^{2} \chi=0 .
$$

If we neglect the time dependence in $m_{k} l e^{-\alpha_{0}}$, the density perturbation (47) satisfies Eq. (48). Thus we found that the existence of $\delta \rho_{\chi}$ depends on the behavior of the bulk perturbations. At low energies, they should have imaginary mass $m_{k}=\sqrt{2 / 3} \mathbf{k} i$ and diverge at the horizon of the AdS spacetime $\left(z=l e^{-\alpha(y, t)} \rightarrow \infty\right)$ because $Z_{2}^{(\chi)}\left(m_{k} z\right)$ contains $H_{2}^{(2)}\left(m_{k} z\right)$ which is proportional to $\exp (\sqrt{2 / 3} \mathbf{k} z)$ for $z \rightarrow \infty$. Thus if we restrict our attention to the bulk perturbations with real mass or with regular behavior in the bulk, the corrections from $\chi$ do not exist on the brane.

Therefore the existence of the corrections from $\chi$ depends on the boundary condition for the perturbations (38). The general solutions for perturbations in the bulk can be written as

$$
\begin{aligned}
h(\mathbf{k})= & e^{-2 \alpha(y, t)} \int d m\left[E^{(1)}(m, \mathbf{k}) Z^{(1)}\left(m l e^{-\alpha(y, t)}\right)\right. \\
& \left.+E^{(2)}(m, \mathbf{k}) Z^{(2)}\left(m l e^{-\alpha(y, t)}\right)\right] e^{-i \omega \tau(y, t)},
\end{aligned}
$$

where $Z^{(1)}$ and $Z^{(2)}$ are two independent combinations of the Hankel functions of the first and second kinds. $E^{(1)}(m, \mathbf{k})$ and $E^{(2)}(m, \mathbf{k})$ are arbitrary coefficients which should be determined by the boundary conditions. One of the choices is the boundary condition that allows the existence of the corrections $\delta \rho_{\chi}$. We choose $E^{(2)}(m, \mathbf{k})$ and $Z^{(2)}$ so that the perturbations contribute to the matter perturbations and do not contribute to the metric perturbations. For example, at low energies, we can take

$$
\begin{aligned}
Z^{(1)}\left(m l e^{-\alpha}\right) & =H^{(1)}\left(m l e^{-\alpha}\right), \\
Z^{(2)}\left(m l e^{-\alpha}\right) & =Z^{(\chi)}\left(m_{k} l e^{-\alpha}\right), \\
E^{(2)}(m, \mathbf{k}) & =E^{(\chi)}(\mathbf{k}) .
\end{aligned}
$$

Then the metric perturbations and the density perturbation induced by these perturbations are given by

$$
\begin{aligned}
\frac{\kappa^{2}}{2 l} \delta \rho= & -3\left(\dot{\alpha}_{0} \dot{\Psi}_{0}+\dot{\alpha}_{0}^{2} \Phi_{0}\right)-e^{-2 \alpha_{0}} \mathbf{k}^{2} \Psi_{0} \\
& +\frac{1}{3} e^{-4 \alpha_{0} \int d m E^{(1)}(m, \mathbf{k}) \mathbf{k}^{4} l^{2} H_{0}^{(1)}\left(m l e^{-\alpha_{0}}\right) e^{i \omega \eta}} \\
& +\frac{\kappa^{2}}{2 l} \delta \rho_{\chi},
\end{aligned}
$$$$
\Psi_{0}(\mathbf{k})=\int d m E^{(1)}(m, \mathbf{k})\left(m l e^{-\alpha_{0}} H_{1}^{(1)}\left(m l e^{-\alpha_{0}}\right)\right.
$$$$
\left.+\frac{1}{3}\left(\mathbf{k} l e^{-\alpha_{0}}\right)^{2} H_{0}^{(1)}\left(m l e^{-\alpha_{0}}\right)\right) e^{i \omega \eta},
$$

where

$$
\begin{aligned}
\delta \rho_{\chi} & =e^{-4 \alpha_{0}} \chi, \\
\chi & =\frac{2 l}{3 \kappa^{2}}\left[\mathbf{k}^{4} l^{2} E^{(\chi)}(\mathbf{k})\right] Z_{0}^{(\chi)}\left(m_{k} l e^{-\alpha_{0}}\right) e^{i \mathbf{k} \eta / \sqrt{3}} .
\end{aligned}
$$

Another choice is the boundary condition that the perturbations are outgoing at the horizon of the AdS spacetime $[19,28,34]$. Then we should take

$$
\begin{aligned}
Z^{(1)}\left(m l e^{-\alpha}\right) & =H^{(1)}\left(m l e^{-\alpha}\right), \\
E^{(2)}(m, \mathbf{k}) & =0 .
\end{aligned}
$$

Note that for imaginary mass $m=i m_{I}, m_{I}>0$, this condition implies that the perturbations do not diverge at the horizon of the AdS spacetime because $H^{(1)}\left(i m_{I} z\right) \propto \exp \left(-m_{I} z\right)$ at $z$ $\rightarrow \infty$. Hence if we take the boundary condition that the perturbations are outgoing, the corrections given by $\chi$ are not allowed:

$$
\delta \rho_{\chi}=\delta p_{\chi}=v_{\chi}=0
$$

The matter perturbations and the metric perturbations are given by Eqs. (40) and (41) with $E(m, \mathbf{k})=E^{(1)}(m, \mathbf{k})$ and $Z\left(m l e^{-\alpha_{0}}\right)=H^{(1)}\left(m l e^{-\alpha_{0}}\right)$.

It seems difficult to determine what kind of perturbations are allowed in the bulk. We will discuss the effects of the corrections from $\chi$ separately according to the choice of the 
boundary condition in Sec. V. For a while we take the boundary condition (53) and then $\delta \rho_{\chi}=\delta p_{\chi}=v_{\chi}=0$. The terms containing $E^{(1)}(m, \mathbf{k})$ in the matter perturbations (40) correspond to the corrections written using the gradient of the metric perturbations in Eqs. (27) and (28). In fact, if we take $\mathbf{k} \rightarrow 0$, the terms containing $E^{(1)}(m, \mathbf{k})$ in the matter perturbations vanish. Now, from Eqs. (40) and (41), we observe that the bulk perturbations alter the relation $\Psi_{0}=\Phi_{0}$ and induce corrections to the matter perturbations. In Eq. (49), $E^{(1)}(m, \mathbf{k})$ is still an arbitrary coefficient. The coefficient $E^{(1)}(m, \mathbf{k})$ can be determined once we impose the equation of state of the matter perturbations, such as $\delta p=c_{s}^{2} \delta \rho$ where $c_{s}^{2}$ is the sound velocity. However, it is difficult to solve the equations for $E^{(1)}(m, \mathbf{k})$. In the following section, we try to obtain the evolution of the perturbations without solving $E^{(1)}(m, \mathbf{k})$. The price to pay is that we must make an assumption about the contribution from the massive modes as in [19]. Recently Gorbunov et al. have shown that the creation of heavy gravitons is negligible in the inflationary brane world [31]. Thus we will assume that the modes with $m l e^{-\alpha_{0}}>1$ do not contribute to the perturbations in the bulk. More precisely, we take

$$
m l e^{-\alpha_{0}} \rightarrow 0 .
$$

From the effective Einstein equations (40) and (41), we find that there are two situations in which the deviation from conventional $4 \mathrm{D}$ theory becomes large. One is given by

$$
\mathbf{k} l e^{-\alpha_{0} \gg 1},
$$

which means that the physical scale of the perturbations is smaller than the curvature scale $l$. This is reasonable since the gravity behaves like that in 5D spacetime at scales smaller than $l$. The other is given by

$$
\dot{\alpha}_{0} l \gg 1,
$$

which means that the energy density of the matter exceeds the tension of the brane. In the Friedmann equation (16), the term proportional to $\rho^{2}$ becomes dominant and the evolution of the universe changes significantly.

\section{MODIFICATIONS OF THE EVOLUTION}

In this section, we take the boundary condition that the perturbations are outgoing at the horizon of the AdS spacetime (53). Then we have

$$
\delta \rho_{\chi}=\delta p_{\chi}=v_{\chi}=0 .
$$

In the following sections, we assume that the matter perturbations are adiabatic.

\section{A. Evolution at superhorizon scales}

Let us consider the long-wavelength perturbations. We take

$$
\mathbf{k} l e^{-\alpha_{0}} \rightarrow 0
$$

then the corrections to the matter perturbations in Eq. (40) written using $E^{(1)}(m, \mathbf{k})$ vanish. From Eq. (40), the evolution equation for the metric perturbations can be obtained by imposing $\delta p-c_{s}^{2} \delta \rho=0$. The equation can be simplified using the Bardeen parameter

$$
\zeta=\Psi_{0}-\frac{\dot{\alpha}_{0}^{2}}{\ddot{\alpha}_{0}}\left(\frac{1}{\dot{\alpha}_{0}} \dot{\Psi}_{0}+\Phi_{0}\right) .
$$

At superhorizon scales $\mathbf{k} \dot{\alpha}_{0}^{-1} e^{-\alpha_{0} \ll 1, \delta p-c_{s}^{2} \delta \rho=0}$ can be written as

$$
\dot{\zeta}=0,
$$

where we used

$$
\dot{w}=-3 \dot{\alpha}_{0}(1+w)\left(c_{s}^{2-w}\right) .
$$

Then the Bardeen parameter is conserved even in the high energy regime, namely,

$$
\zeta=\zeta_{*}=\text { const. }
$$

We should note that the constancy of the Bardeen parameter does not mean that the behavior of the perturbations in the brane world is the same as that obtained in conventional 4D theory. The Bardeen parameter is written in terms of $\Phi_{0}$ and $\Psi_{0}$. In conventional $4 \mathrm{D}$ theory we have the equation $\Phi_{0}$ $=\Psi_{0}$. In the brane world, however, it is modified by the perturbations in the bulk. An equation that gives the relation between $\Psi_{0}$ and $\Phi_{0}$ is needed. From Eq. (41), the metric perturbations are given by

$$
\begin{aligned}
\Psi_{0}= & \int d m E^{(1)}(m) m l e^{-\alpha_{0}} H_{1}^{(1)}\left(m l e^{-\alpha_{0}}\right) e^{-i m T}, \\
\Phi_{0}= & {\left[1+\left(\dot{\alpha}_{0} l\right)^{2}\right] \Psi_{0}-\left[1+2\left(\dot{\alpha}_{0} l\right)^{2}\right] \int d m E^{(1)}(m) } \\
& \times\left(m l e^{-\alpha_{0}}\right)^{2} H_{0}^{(1)}\left(m l e^{-\alpha_{0}}\right) e^{-i m T} \\
& -2 i \alpha_{1} \dot{\alpha}_{0} l^{2} \int d m E^{(1)}(m) \\
& \times\left(m l e^{-\alpha_{0}}\right)^{2} H_{1}^{(1)}\left(m l e^{-\alpha_{0}}\right) e^{-i m T} .
\end{aligned}
$$

As mentioned in the previous section, we should make some assumption about the contribution from massive modes. We will assume that the modes with $m l e^{-\alpha_{0}}>1$ do not contribute to the perturbations in the bulk and thus take

$$
m l e^{-\alpha_{0}} \rightarrow 0 .
$$

Then using the asymptotic forms of the Hankel functions $H_{1}^{(1)}(z) \propto 1 / z$ and $H_{0}^{(1)}(z) \propto$ const, we obtain

$$
\Phi_{0}=\left[1+\left(\dot{\alpha}_{0} l\right)^{2}\right] \Psi_{0} .
$$

At high energy, we have

$$
\Phi_{0}=\left(\dot{\alpha}_{0} l\right)^{2} \Psi_{0} ;
$$


thus $\Phi_{0} \gg \Psi_{0}$. From the conservation of the Bardeen parameter Eqs. (60) and (63), we get

$$
\begin{aligned}
& \Phi_{0}=3(1+w) \zeta_{*}, \quad \Psi_{0}=\left(\dot{\alpha}_{0} l\right)^{-2} \Phi_{0}, \\
& \frac{\delta \rho}{\rho}=-\Phi_{0},
\end{aligned}
$$

for $w=$ const. Note that the curvature perturbation increases as $\Psi_{0} \propto \rho^{-2}$ at high energy.

At low energy, $\dot{\alpha}_{0} l \ll 1$, we have

$$
\Psi_{0}=\Phi_{0}
$$

Then the metric perturbations are obtained as

$$
\begin{aligned}
& \Phi_{0}=\Psi_{0}=\frac{3(1+w)}{5+3 w} \zeta_{*}, \\
& \frac{\delta \rho}{\rho}=-2 \Phi_{0},
\end{aligned}
$$

for $w=$ const.

The CMB anisotropies at large scales can be obtained using the above solutions. At the decoupling of the photon and baryon, the energy of the universe is lower than the tension of the brane $\dot{\alpha}_{0} l \ll 1$. The temperature anisotropies caused by the ordinary Sachs-Wolfe effect are given by

$$
\frac{\Delta T}{T}=\frac{1}{4} \frac{\delta \rho_{r}}{\rho_{r}}+\Phi_{0}=\frac{1}{3} \frac{\delta \rho}{\rho}+\Phi_{0},
$$

where $\rho_{r}$ is the density of the radiation and $\delta \rho_{r}$ is its perturbation. From Eqs. (40) and (60), we can show the Bardeen parameter is given by

$$
\zeta=\Psi_{0}-\frac{1}{3} \frac{\delta \rho}{\rho} .
$$

Then the temperature anisotropies can be evaluated as

$$
\frac{\Delta T}{T}=-\zeta+\Psi_{0}+\Phi_{0}
$$

If we neglect the effect of the massive graviton with $m l e^{-\alpha_{0}}>1$, we can evaluate the temperature anisotropies as

$$
\frac{\Delta T}{T}=\frac{1}{5} \zeta_{*},
$$

where we used the solution (70) with $w=0$.

The massive graviton will modify the relation between $\Phi_{0}$ and $\Psi_{0}$ and thus the temperature anisotropies. At low energy, the metric perturbations are given by

$$
\begin{aligned}
\Psi_{0}= & \int d m E^{(1)}(m) m l e^{-\alpha_{0}} H_{1}^{(1)}\left(m l e^{-\alpha_{0}}\right) e^{i m \eta}, \\
\Phi_{0}= & \Psi_{0}-\int d m E^{(1)}(m) \\
& \times\left(m l e^{-\alpha_{0}}\right)^{2} H_{0}^{(1)}\left(m l e^{-\alpha_{0}}\right) e^{i m \eta} .
\end{aligned}
$$

Then at the lowest order corrections in $m l e^{-\alpha_{0}}$ we have

$$
\Phi_{0}=\Psi_{0}-\int d m \Psi_{0}(m)\left(m l e^{-\alpha_{0}}\right)^{2} G_{K K}\left(m l e^{-\alpha_{0}}\right) e^{i m \eta}
$$

Here $\Psi_{0}(m)$ denotes the Fourier transformation of $\Psi_{0}(\eta)$ with respect to $\eta$ and

$$
\begin{aligned}
G_{K K}\left(m l e^{-\alpha_{0}}\right) & =\lim _{m l e^{-\alpha_{0} \rightarrow 0}}\left(\frac{H_{0}^{(1)}\left(m l e^{-\alpha_{0}}\right)}{m l e^{-\alpha_{0}} H_{1}^{(1)}\left(m l e^{-\alpha_{0}}\right)}\right) \\
& =\ln \left(2 e^{\alpha_{0}}\right)-\gamma+\frac{\pi}{2} i-\ln (m l) .
\end{aligned}
$$

Here $\gamma$ is the Euler number. The important point is that $G_{K K}$ contains a nonanalytic term proportional to $\ln m$. Thus Eq. (76) becomes nonlocal when we make a Fourier transformation to real spacetime. The reason can be understood as follows. The massive modes with $m \neq 0$ can propagate into the bulk. These modes affect the metric perturbations nonlocally if they are observed on the brane. Thus the nonlocality of the evolution equation is an essential feature of the brane world [20].

The contributions from the massive modes are determined by $E^{(1)}(m)$, which is determined by the primordial fluctuations and later evolution. It is difficult to know $E^{(1)}(m)$, but it should be noted that in Eq. (76) $m$ appears in the form $m l e^{-\alpha_{0}}$. Thus, as the energy of the universe becomes lower $e^{-\alpha_{0}} \rightarrow 0$, the mass of the massive modes that can modify the relation $\Phi_{0}=\Psi_{0}$ becomes larger. Then for late times we can safely use the standard result (74). The constant $\zeta_{*}$ should be determined by the primordial fluctuations. We discuss the generation of the primordial fluctuations in Appendix C.

Here is a point we should emphasize. At high energy, the Hubble scale itself is smaller than the curvature scale of the AdS spacetime. Thus we should be careful in using the result $\mathbf{k} l e^{-\alpha_{0}} \rightarrow 0$ even at superhorizon scales in the high energy universe.

\section{B. Evolution at subhorizon scales}

In this section we investigate the corrections that arise for

$$
\mathbf{k} l e^{-\alpha_{0}} \neq 0 \text {. }
$$

We will assume the universe is in the low energy era $\dot{\alpha}_{0} l$ $\ll 1$ and take

$$
m l e^{-\alpha_{0}} \rightarrow 0 .
$$


At subhorizon scales $\mathbf{k} \dot{\alpha}_{0}^{-1} e^{-\alpha_{0} \gg 1}$, the density perturbation (40) is given by

$$
\begin{aligned}
\frac{\kappa^{2}}{2 l} e^{2 \alpha_{0}} \delta \rho= & -\mathbf{k}^{2} \Psi_{0}+\frac{1}{3} e^{-2 \alpha_{0}} \\
& \times \int d m E^{(1)}(m, \mathbf{k}) \mathbf{k}^{4} l^{2} H_{0}^{(1)}\left(m l e^{-\alpha_{0}}\right) e^{i \omega \eta} \\
= & -\int d m E^{(1)}(m, \mathbf{k}) \mathbf{k}^{2} m l e^{-\alpha_{0}} H_{1}^{(1)}\left(m l e_{0}^{-\alpha}\right) e^{i \omega \eta} .
\end{aligned}
$$

On the other hand the metric perturbations (41) are given by

$$
\begin{aligned}
\mathbf{k}^{2} \Phi_{0}= & \int d m E^{(1)}(m, \mathbf{k}) \mathbf{k}^{2}\left(m l e^{-\alpha_{0}} H_{1}^{(1)}\left(m l e^{-\alpha_{0}}\right)\right. \\
& \left.-\frac{1}{3}\left(\mathbf{k}^{2}+3 m^{2}\right) l^{2} e^{-2 \alpha_{0}} H_{0}^{(1)}\left(m l e^{-\alpha_{0}}\right)\right) e^{i \omega \eta} .
\end{aligned}
$$

For $m l e^{-\alpha_{0} \ll 1}$ we can rewrite Eq. (81) into the effective Poisson equation:

$$
\begin{aligned}
\mathbf{k}^{2} \Phi_{0}= & -\frac{\kappa^{2}}{2 l} e^{2 \alpha_{0}} \delta \rho+\frac{\kappa^{2} l}{6} e^{-2 \alpha_{0}} \int d m\left(\mathbf{k}^{2}+3 m^{2}\right) \\
& \times G_{K K}\left(m l e^{-\alpha_{0}}\right)\left[e^{2 \alpha_{0}} \delta \rho\right](m, \mathbf{k}),
\end{aligned}
$$

where $G_{K K}$ is given by Eq. (77) and $\left[e^{2 \alpha_{0}} \delta \rho\right](m, \mathbf{k})$ denotes the Fourier transformation of $e^{2 \alpha_{0}} \delta \rho$ with respect to $\eta$ and $x^{i}$. For $\mathbf{k} l e^{-\alpha_{0}} \rightarrow 0$, Eq. (82) is the usual Poisson equation. The evolution equation for $\delta \rho$ can be derived from the conservation laws of the matter perturbations (20) and (21). For example, in the matter dominated era $w=0$, we get

$$
\begin{gathered}
\Delta^{\prime \prime}+\alpha_{0}{ }^{\prime} \Delta^{\prime}-\frac{3}{2} \alpha_{0}^{\prime 2} \Delta+\frac{\kappa^{2} l}{6} e^{-2 \alpha_{0}} \int d m\left(\mathbf{k}^{2}+3 m^{2}\right) \\
\times G_{K K}\left(m l e^{-\alpha_{0}}\right)\left[e^{2 \alpha_{0}} \delta \rho\right](m, \mathbf{k}) e^{-i \omega \eta}=0,
\end{gathered}
$$

where the prime denotes the derivative with respect to $\eta$ and $\Delta=\delta \rho / \rho$. The last term represents the correction from the bulk perturbations. Note that for $\mathbf{k} l e^{-\alpha_{0}} \neq 0$ a nonlocal term arises even if we take $m l e^{-\alpha_{0}} \rightarrow 0$. This is because the graviton can easily propagate into the bulk at scales smaller than $l\left(\mathbf{k} l e^{-\alpha_{0}}>1\right)$. Thus the bulk gravitational field affects the evolution of the density perturbation nonlocally.

It is well known that in the Minkowski brane Newton's law is modified due to the $5 \mathrm{D}$ graviton $[3,32-34]$ This modification can be derived from the effective Poisson equation. Let us consider the situation where $e^{\alpha_{0}}=1$. We assume that the source is static $\omega^{2}=m^{2}+\mathbf{k}^{2}=0$, and derive the lowest order corrections in $(\mathbf{k} l)^{2}<1$. Taking the nonanalytic term, the metric perturbations are written as

$$
\begin{aligned}
& \Psi_{0}(\mathbf{k})=-\frac{\kappa^{2}}{2 l}\left(\mathbf{k}^{-2}-\frac{1}{3} l^{2} \ln (m l)\right) \delta \rho(\mathbf{k}), \\
& \Phi_{0}(\mathbf{k})=-\frac{\kappa^{2}}{2 l}\left(\mathbf{k}^{-2}-\frac{2}{3} l^{2} \ln (m l)\right) \delta \rho(\mathbf{k}) .
\end{aligned}
$$

To compare the result with the one obtained in [31-33], we consider a spherically symmetric source and derive the metric perturbations far away from the source. We obtain the metric perturbations by Fourier transformation as

$$
\begin{aligned}
& \Psi_{0}(r)=-\frac{G_{4} M}{r}\left(1+\frac{l^{2}}{3 r^{2}}\right), \\
& \Phi_{0}(r)=-\frac{G_{4} M}{r}\left(1+\frac{2 l^{2}}{3 r^{2}}\right),
\end{aligned}
$$

where $8 \pi G_{4}=\kappa^{2} / l, M=\int d \mathbf{x}^{3} \delta \rho(x)$, and the source is located at $r=0$. This result completely agrees with the one obtained in [31-33].

\section{CORRECTIONS FROM PERTURBATIONS OF DARK RADIATION}

In this section we choose the boundary condition so that the corrections $\delta \rho_{\chi}, \delta p_{\chi}$, and $v_{\chi}$ are induced on the brane. Then we investigate the effects of the corrections on the evolution of the perturbations.

\section{A. Evolution at superhorizon scales}

Let us consider the long-wavelength perturbations with $\mathbf{k} l e^{-\alpha_{0}} \rightarrow 0$. The corrections to the matter perturbations given by $E^{(1)}(m, \mathbf{k})$ vanish. At superhorizon scales $\mathbf{k} \dot{\alpha}_{0}^{-1} e^{-\alpha_{0} \ll 1}$, the density perturbation and the pressure perturbation are obtained from Eqs. (29) and (40) as

$$
\begin{aligned}
-\frac{\kappa^{2} \alpha_{1}}{2} \delta \rho= & -3\left(\dot{\alpha}_{0} \dot{\Psi}_{0}+\dot{\alpha}_{0}^{2} \Phi_{0}\right)+\frac{\kappa^{2}}{2 l} C e^{-4 \alpha_{0},} \\
-\frac{\kappa^{2} \alpha_{1}}{2} \delta p= & \ddot{\Psi}_{0}+\left(3 \dot{\alpha}_{0}-\frac{\dot{\alpha}_{0} \ddot{\alpha}_{0}}{\alpha_{1}^{2}}\right) \dot{\Psi}_{0}+\dot{\alpha}_{0} \dot{\Phi}_{0} \\
& +\left(2 \ddot{\alpha}_{0}-\frac{\dot{\alpha}_{0}^{2} \ddot{\alpha}_{0}}{\alpha_{1}^{2}}+3 \dot{\alpha}_{0}^{2}\right) \Phi_{0} \\
& +\frac{\kappa^{2}}{2 l} C e^{-4 \alpha_{0}} \frac{1}{3}\left(1+\frac{\ddot{\alpha}_{0}}{\alpha_{1}^{2}}\right),
\end{aligned}
$$

where we put $\chi=C=$ const, which can be deduced from Eq. (26). Then, using the Bardeen parameter (60), $\delta p-c_{s}^{2} \delta \rho$ $=0$ can be written as

$$
\dot{\zeta}=\frac{\kappa^{2}}{2 l} C e^{-4 \alpha_{0}} \frac{\dot{\alpha}_{0}}{\ddot{\alpha}_{0}}\left[\frac{1}{3}\left(1+\frac{\ddot{\alpha}_{0}}{\alpha_{1}^{2}}\right)-c_{s}^{2}\right] .
$$

We see that the term proportional to $C$ breaks the constancy of the Bardeen parameter. The results can be understood as follows. The density perturbations $\delta \rho_{\chi}$ induce isocurvature 
perturbations on the brane. In $4 \mathrm{D}$ theory, it is well known that the isocurvature perturbations break the constancy of the Bardeen parameter. In fact, if we consider the perturbations in the radiation dominated era at low energy

$$
c_{s}^{2}=\frac{1}{3}, \quad \alpha_{1} \gg \dot{\alpha}_{0},
$$

then $\dot{\zeta}=0$. This is reasonable since $\delta \rho_{\chi}$ behaves as radiation $\left[\delta p_{\chi}=(1 / 3) \delta \rho_{\chi}\right]$ at low energies, so there are no isocurvature perturbations. The equation can be integrated using the background equations (A12) and (62). We get

$$
\begin{aligned}
\zeta & =\zeta_{*}-\frac{\kappa^{2}}{2 l} \frac{C e^{-4 \alpha_{0}}}{3 \ddot{\alpha}_{0}} \\
& =\zeta_{*}-\frac{1}{3(1+w)} \frac{1}{l \alpha_{1}}\left(\frac{\rho_{r}}{\rho}\right) C_{*},
\end{aligned}
$$

where we defined

$$
C_{*}=C \frac{e^{-4 \alpha_{0}}}{\rho_{r}}=\text { const. }
$$

Using the expression for the Bardeen parameter in terms of the metric perturbations (60), we can obtain the solutions for the metric perturbations.

At high energy, using

$$
\Psi_{0}=\frac{1}{\left(\dot{\alpha}_{0} l\right)^{2}} \Phi_{0} \ll \Phi_{0},
$$

we get

$$
\Phi_{0}=3(1+w) \zeta_{*}+\frac{1}{\dot{\alpha}_{0} l}\left(\frac{\rho_{r}}{\rho}\right) C_{*}
$$

for $w=$ const. Note that the contribution from $C_{*}$ is suppressed by the factor $\left(\dot{\alpha}_{0} l\right)^{-1}$.

At low energy, using

$$
\Psi_{0}=\Phi_{0}
$$

we get

$$
\Phi_{0}=\frac{3(1+w)}{5+3 w} \zeta_{*}+\frac{1}{3(1+3 w)}\left(\frac{\rho_{r}}{\rho}\right) C_{*}
$$

for $w=$ const.

The CMB anisotropies (73) caused by the ordinary SachsWolfe effect in the matter dominated era are given by

$$
\begin{aligned}
\frac{\Delta T}{T} & =-\zeta+2 \Phi_{0} \\
& =\frac{1}{5} \zeta_{*}+\frac{1}{3}\left(\frac{\rho_{r}}{\rho}\right) C_{*} .
\end{aligned}
$$

From the observations, the magnitude of the anisotropies is known as $\Delta T / T \sim 10^{-5}$ and the fraction of the radiation component in the total density is $\rho_{r} / \rho \sim 0.1$ at decoupling. Then the constraint on $C_{*}$ is obtained as [29]

$$
C_{*}<10^{-4}
$$

\section{B. Evolution at subhorizon scales}

Now consider the evolution of the perturbations at subhorizon scales $\mathbf{k} \dot{\alpha}_{0}^{-1} e^{-\alpha_{0} \gg 1}$. For simplicity, we consider the low energy regime. We also assume that the length scale of the perturbations is larger than $l\left(\mathbf{k} l e^{-\alpha_{0} \ll 1}\right)$. Then the corrections due to $E^{(1)}(m, \mathbf{k})$ can be neglected. In order to describe the evolution of the density perturbation, it is convenient to introduce the gauge invariant variable defined by

$$
\rho \Delta=\delta \rho+3 \dot{\alpha}_{0}(\rho+p) e^{\alpha_{0}} v
$$

From Eqs. (40) and (29) the Poisson equation is given by

$$
\mathbf{k}^{2} \Phi_{0}=-\frac{3}{2} \alpha_{0}^{\prime 2} \Delta+e^{-2 \alpha_{0}} \frac{\kappa^{2}}{2 l}\left(\chi-3 \alpha_{0}^{\prime} \mathbf{k}^{-2} \chi^{\prime}\right) \text {. }
$$

Equations (20) and (21) become

$$
\begin{aligned}
\Delta^{\prime}-3 w \alpha_{0}^{\prime} \Delta & =-(1+w) \mathbf{k}^{2} v+\frac{3 \kappa^{2}}{2 l}(1+w) e^{-2 \alpha_{0}} \mathbf{k}^{-2} \chi^{\prime} \\
v^{\prime}+\alpha_{0}^{\prime} v & =\Phi_{0}+\frac{c_{s}^{2}}{1+w} \Delta
\end{aligned}
$$

where we used the formula (62). Then the evolution equation for $\Delta$ can be obtained as

$$
\begin{gathered}
\Delta^{\prime \prime}-\left[3\left(2 w-c_{s}^{2}\right)-1\right] \alpha_{0}^{\prime} \Delta^{\prime}+3\left(\frac{3}{2} w^{2}-4 w-\frac{1}{2}+3 c_{s}^{2}\right) \alpha_{0}^{\prime 2} \Delta \\
+c_{s}^{2} \mathbf{k}^{2} \Delta=-\frac{\kappa^{2}}{l}(1+w) e^{-2 \alpha_{0}} \chi
\end{gathered}
$$

The initial condition for $\Delta$ can be set in the radiation dominated era. In the radiation dominated era, $w=c_{s}^{2}=1 / 3$ and $e^{\alpha_{0}}=\eta$, the evolution equation (100) becomes

$$
\Delta^{\prime \prime}-\frac{2}{\eta^{2}} \Delta+\frac{1}{3} \mathbf{k}^{2} \Delta=-\frac{4 \kappa^{2}}{3 l} \frac{1}{\eta^{2}} \chi
$$

Then we can easily find the solution as

$$
\Delta=A U_{G}(\eta)+B U_{D}(\eta)+\frac{2 \kappa^{2}}{3 l} \chi
$$

where

$$
U_{G}=-\cos \left(\mathbf{k}_{s} \eta\right)+\left(\frac{1}{\mathbf{k}_{s} \eta}\right) \sin \left(\mathbf{k}_{s} \eta\right)
$$




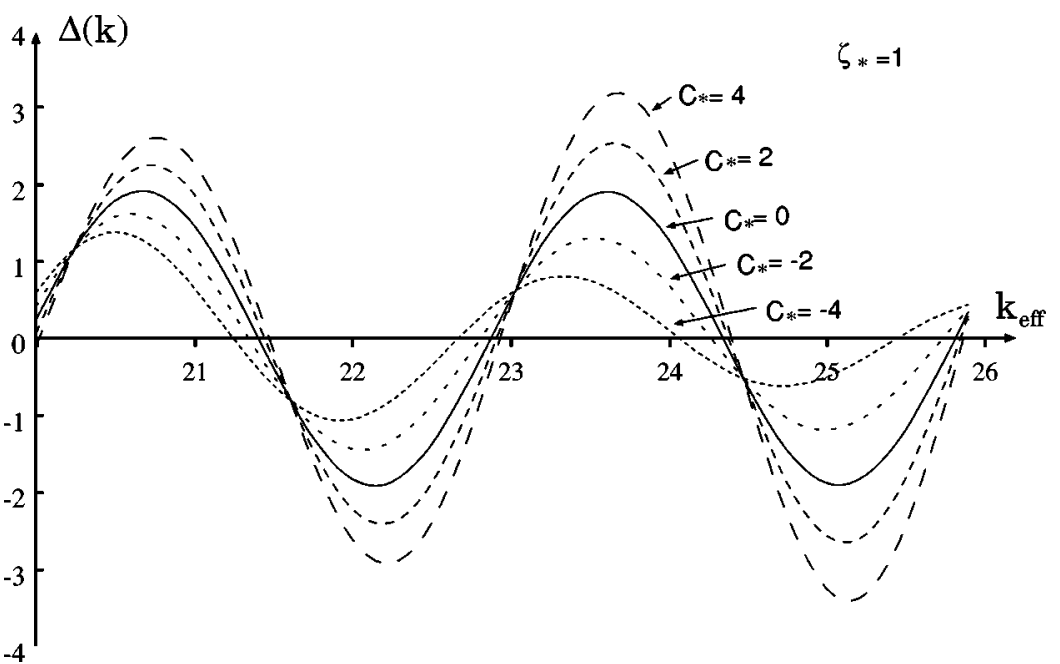

FIG. 1. $\Delta(\mathbf{k})$ at $\rho_{r} / \rho_{m}=0.1$ with $C_{*}=0$, $2 \zeta_{*}, 4 \zeta_{*},-2 \zeta_{*},-4 \zeta_{*}$ where we take $\zeta_{*}$ $=1$. The horizontal coordinate is the value $\mathbf{k}_{e f f}$ $=\dot{\alpha}_{0}^{-1} e^{-\alpha_{0}} \mathbf{k}$ at $\rho_{r} / \rho_{m}=1$. The perturbation with $\mathbf{k}_{\text {eff }}=1$ crosses the horizon at $\rho_{r} / \rho_{m}=1$. The initial conditions are set at $\rho_{r} / \rho_{m}=100$.

$$
U_{D}=-\sin \left(\mathbf{k}_{s} \eta\right)-\left(\frac{1}{\mathbf{k}_{s} \eta}\right) \cos \left(\mathbf{k}_{s} \eta\right)
$$

$A$ and $B$ are constants of integration, and $\mathbf{k}_{s}=\mathbf{k} / \sqrt{3}$. Note that for $\mathbf{k} \eta \rightarrow 0, U_{G}$ and $U_{D}$ behave as $U_{G}=\mathbf{k}^{2} \eta^{2} / 9$ and $U_{D} \propto \eta^{-1}$. Then $U_{G}$ matches the growing mode solution at superhorizon scales. From Eq. (98), the metric perturbation is given by

$$
\Phi_{0}=-\frac{1}{\mathbf{k}^{2} \eta^{2}}\left[\frac{3}{2}\left(A U_{G}+B U_{D}\right)+\frac{\kappa^{2}}{2 l}\left(\chi+\frac{3}{\eta} \mathbf{k}^{-2} \chi^{\prime}\right)\right] .
$$

From Eq. (48), the solution for $\chi$ is given by

$$
\chi=C \cos \left(\mathbf{k}_{s} \eta\right)+D \sin \left(\mathbf{k}_{s} \eta\right),
$$

where $D$ is an arbitrary constant. Then $\Phi_{0}$ becomes

$$
\Phi_{0}=-\frac{1}{\mathbf{k}^{2} \eta^{2}}\left[\left(\frac{3}{2} A-\frac{\kappa^{2}}{2 l} C\right) U_{G}+\left(\frac{3}{2} B-\frac{\kappa^{2}}{2 l} D\right) U_{D}\right] .
$$

We take only the growing mode solution; then $B=D=0$. In the radiation dominated era, we obtain

$$
\alpha_{0}^{\prime 2} e^{-2 \alpha_{0}}=\frac{\kappa^{2}}{3 l} \rho_{r},
$$

and $e^{\alpha_{0}}=\eta$. Then Eq. (90) becomes

$$
C_{*}=\frac{\kappa^{2}}{3 l} C
$$

At superhorizon scales, using $U_{G}=\mathbf{k}^{2} \eta^{2} / 9$, we get

$$
\Phi_{0}=-\frac{A}{6}+\frac{1}{6} C_{*} .
$$

Comparing the solution Eq. (109) with (94), we find $A=$ $-4 \zeta_{*}$. Thus, we can set the initial condition for $\Delta$ in the radiation dominated era as

$$
\Delta=-4 \zeta_{*} U_{G}+2 C_{*} \cos \left(\mathbf{k}_{s} \eta\right) .
$$

In the radiation dominated era, the perturbations are constant $\Delta \sim 2 C_{*}$ at superhorizon scales and then oscillate as a cosine function once they enter the horizon. Thus, at subhorizon scales, the density perturbation behaves like the usual adiabatic perturbations in 4D theory. However, as the matter becomes dominant, isocurvature perturbations are generated. This is because, while the frequency of $U_{G}$ changes from $\mathbf{k}_{s}$, $\chi$ always oscillates with frequency $\mathbf{k}_{s}$. Thus there is a possibility that the amplitude and phase of the oscillations of $\Delta$ change from the adiabatic cosine mode. These deviations can be directly observed as the shifts of the location and height of the peak of the acoustic oscillation in the CMB spectrum. We solved Eq. (100) numerically with the initial condition given by Eq. (110). In Fig. 1, the density perturbation $\Delta(\mathbf{k})$ at the time $\rho_{r} / \rho_{m}=0.1$ is shown with various wave numbers k. Here $\rho_{m}$ is the density of matter. For $C_{*}=0, \Delta(\mathbf{k})$ is given by a cosine function. If we include the effect of $C_{*}$, the location and height of the peak of the oscillations change as expected. Thus, if we include the effect of the corrections $\delta \rho_{\chi}, \delta p_{\chi}$, and $v_{\chi}$, the effects from the bulk can be observed even in the low energy universe.

\section{CONCLUSION}

In this paper we obtained the effective 4D Einstein equations (40) and (41) that describe the scalar cosmological perturbations on the brane. Then we investigated the effect of the bulk gravitational field on the evolution of the cosmological perturbations on the brane.

We first used the equations on the brane obtained from a power series expansion of the 5D Einstein equations. From the equations on the brane, we obtained the effective Einstein equations (27), (28), and (29). It should be mentioned that we cannot derive the equation that contains only the variables on the brane and gives the relation between the metric perturbations $\Phi_{0}$ and $\Psi_{0}$. Two types of correction are found. One is given by the gradient of the metric perturbations. The other is independent of the metric perturbations (29) and induces density perturbations, which behave like sound 
waves with the sound velocity $1 / \sqrt{3}$ at low energy. At large scales, they are homogeneous perturbations that depend only on time and decay like radiation. We identified them with the perturbations of the dark radiation.

Then we derived the effective Einstein equations again in another way by solving the perturbations in the bulk and imposing the junction conditions (40) and (41). We obtained an equation that gives the relation between $\Phi_{0}$ and $\Psi_{0}$. We identified the perturbations in the bulk that induce perturbations of the dark radiation. These perturbations do not contribute to the metric perturbations but do contribute to the matter perturbations on the brane. At low energy, they have imaginary mass $2 \mathbf{k}^{2}+3 m^{2}=0$ in the bulk and diverge at the horizon of the AdS spacetime. Their existence in the bulk depends on the boundary conditions of the perturbations. We should impose two boundary conditions to completely determine the perturbations in the bulk. One is given by the equation of state of the matter on the brane. The other choice of boundary condition at the horizon determines the existence of the perturbations of the dark radiation.

If we take the boundary condition that the perturbations do not diverge at the horizon of the AdS spacetime, the perturbations of the dark radiation do not appear. The other corrections are suppressed by $\mathbf{k} l e^{-\alpha_{0}}$. Thus they correspond to correction terms given by the gradient of the metric perturbations. Corrections also arise in the relation between $\Phi_{0}$ and $\Psi_{0}$. The corrections become large at scales smaller than the curvature scales of the AdS spacetime $\left(\mathbf{k} l e^{-\alpha_{0} \gg 1}\right)$ and in the high energy universe with energy density larger than the tension of the brane $\left(\dot{\alpha}_{0} l \gg 1\right)$. In particular, at high energy $\dot{\alpha}_{0} l \gg 1$, the potential perturbation $\Phi_{0}$ becomes dominant over the curvature perturbation $\Psi_{0}$. We discuss the evolution of the adiabatic perturbations including these corrections. The interesting point is that at sufficiently large scales $\left(\mathbf{k} l e^{-\alpha_{0}} \rightarrow 0\right)$ the Bardeen parameter is constant even at high energies. Then the potential perturbations $\Phi_{0}$ are always constant if the barotropic parameter of the matter $w$ is constant. On the contrary, at scales below $l$, the correction becomes large. In order to illustrate how these corrections modify the evolution of the density perturbations, we obtained the effective Poisson equation in the low energy universe at subhorizon scales. Using the effective Poisson equation, the evolution equation for the matter perturbations was given. The important point is that the evolution equation becomes nonlocal once we incorporate the effect of the perturbations in the bulk. This is the essential feature of the perturbations at scales below $l$. We emphasized that one should be careful to use the result $\mathbf{k} l e^{-\alpha_{0}} \rightarrow 0$ in the high energy universe even at superhorizon scales. This is because at high energy the horizon scale of the universe itself is smaller than the curvature scale $l$.

We should comment on our limitation in obtaining the evolution of the perturbations using the effective Einstein equations. It is in general difficult to obtain the spectrum $E^{(1)}(m, \mathbf{k})$ of the perturbations in the bulk by imposing the equation of state of the matter perturbations. As a result, we should make an assumption about the contribution of the massive perturbations. We used the assumption that the modes with $m l e^{-\alpha_{0}}>1$ do not contribute to the perturbations in the bulk; thus we take $m l e^{-\alpha_{0}} \rightarrow 0$. At low energy $e^{-\alpha_{0}} \rightarrow 0$, the assumption seems to be valid. The coefficient $E^{(1)}(m, \mathbf{k})$ is determined by the primordial fluctuations and later evolution [31,36,37]. Further studies are needed to know the exact form of $E^{(1)}(m, \mathbf{k})$.

If we choose appropriate boundary conditions in the bulk (50), perturbations of the dark radiation arise. They induce isocurvature perturbations in the dust dominated universe. Their key feature is that they can play a role even in the low energy universe at scales larger than $l$ where the previous corrections are suppressed. We gave an evolution equation for the density perturbation including the corrections from them. The large scale CMB anisotropies were estimated and the constraint on the amplitude was derived. At subhorizon scales, they act as an extra force on the acoustic oscillations of the density perturbation. In the matter dominated era, the location and the height of the acoustic peak are shifted due to the extra force (see Fig. 1). These shifts can be directly observed by CMB anisotropies. Recently, much work has been done to test the correlation between adiabatic and isocurvature perturbations using the CMB spectrum [35]. Detailed analysis of the CMB spectrum will reveal the existence of the dark sound waves.

\section{ACKNOWLEDGMENTS}

The work of K. K. was supported by JSPS.

\section{APPENDIX A: BACKGROUND EQUATIONS}

In this Appendix, we derive the equations used in Sec. II. The 5D Einstein equations are given by

$$
\begin{aligned}
G_{N}^{M} & =\frac{6}{l^{2}} \delta_{N}^{M}+\kappa^{2} \frac{\sqrt{-g_{\text {brane }}}}{\sqrt{-g}} T_{N}^{M} \\
& =\frac{6}{l^{2}} \delta_{N}^{M}+e^{-\beta} \kappa^{2} T_{N}^{M} \quad\left(M, N=y, t, x^{i}\right) .
\end{aligned}
$$

We take the energy-momentum tensor in the 5D spacetime as

$$
T_{N}^{M}=\left(-\frac{6}{\kappa^{2} l} \operatorname{diag}(0,1,1,1,1)+\operatorname{diag}(0,-\rho, p, p, p)\right) \delta(y) .
$$

The Einstein tensor is given by

$$
\begin{aligned}
& G_{0}^{0}=-3 e^{-2 \beta}\left(\dot{\alpha}^{2}+\dot{\alpha} \dot{\beta}-\alpha^{\prime \prime}-2 \alpha^{\prime 2}+\alpha^{\prime} \beta^{\prime}\right), \\
& G_{y}^{y}=3 e^{-2 \beta}\left(-\ddot{\alpha}-2 \dot{\alpha}^{2}+\dot{\alpha} \dot{\beta}+\alpha^{\prime 2}+\alpha^{\prime} \beta^{\prime}\right), \\
& G_{y}^{0}=-3 e^{-2 \beta}\left(\beta^{\prime} \dot{\alpha}+\alpha^{\prime} \dot{\beta}-\dot{\alpha}^{\prime}-\dot{\alpha} \alpha^{\prime}\right), \\
& G_{j}^{i}=\delta_{j}^{i} e^{-2 \beta}\left(-2 \ddot{\alpha}-3 \dot{\alpha}^{2}-\ddot{\beta}+2 \alpha^{\prime \prime}+3 \alpha^{\prime 2}+\beta^{\prime \prime}\right) .
\end{aligned}
$$

In the $(0,0)$ and $(i, j)$ components of the Einstein equations, the jump of the first derivative of $\alpha(y, t)$ and $\beta(y, t)$ gives 
the $\delta(y)$ function. This should be equated with the $\delta(y)$ function of the matter. Thus we obtain the junction conditions (7)

$$
\begin{aligned}
& \alpha_{1}(t)=-\frac{1}{l}-\frac{\kappa^{2} \rho(t)}{6}, \\
& \beta_{1}(t)=-\frac{1}{l}+\frac{\kappa^{2} \rho(t)}{3}+\frac{\kappa^{2} p(t)}{2},
\end{aligned}
$$

where we take $e^{\beta_{0}}=1$. The $y^{0}$ th order of the $(y, y)$ and $(y, 0)$ components of the Einstein equations gives

$$
\begin{aligned}
-\ddot{\alpha}_{0}-2 \dot{\alpha}_{0}^{2}+\alpha_{1}^{2}+\alpha_{1} \beta_{1} & =\frac{2}{l^{2}}, \\
\beta_{1} \dot{\alpha}_{0}-\dot{\alpha}_{1}-\dot{\alpha}_{0} \alpha_{1} & =0 .
\end{aligned}
$$

Using the junction condition (A4), we get Eq. (8).

Next let us derive the wave equations for $\beta$ and $\alpha$ in the bulk. The $(0,0)$ and $(i, j)$ components of the 5D Einstein equations in the bulk can be rewritten using $u=(t-y) / l$ and $v=(t+y) / l$ as

$$
\begin{aligned}
\alpha_{, u v}+3 \alpha_{, u} \alpha_{, v}+e^{2 \beta} & =0, \\
\beta_{, u v}-3 \alpha_{, u} \alpha_{, v}-\frac{1}{2} e^{2 \beta} & =0 .
\end{aligned}
$$

We assume that the bulk is AdS spacetime, so we take $C_{M y N y}=0$ where $C_{M N K L}$ is the Weyl tensor. This condition is given by

$$
\alpha_{, u v}-\beta_{, u v}=0 .
$$

Then the wave equations for $\alpha$ and $\beta$ become

$$
\begin{aligned}
& \beta_{, u v}+\frac{1}{4} e^{2 \beta}=0, \\
& \alpha_{, u v}+\frac{1}{4} e^{2 \beta}=0 .
\end{aligned}
$$

Thus we derived Eq. (12) in Sec. II. Instead of solving the wave equations directly, it is convenient to rewrite the equation for $\alpha$ using Eq. (A6) as

$$
\alpha_{, u v}-\alpha_{, u} \alpha_{, v}=0
$$

The solution can be found easily as

$$
e^{\alpha}=\frac{1}{f(u)-g(v)},
$$

where $f(u)$ and $g(v)$ are arbitrary functions. Then $\beta$ can be obtained from Eq. (A6) as

$$
\begin{aligned}
e^{2 \beta} & =-\alpha_{, u v}-3 \alpha_{, u} \alpha_{, v} \\
& =4 \frac{f^{\prime}(u) g^{\prime}(v)}{[f(u)-g(v)]^{2}} .
\end{aligned}
$$

Finally, we show some background equations that are used in the calculations of the perturbations. From the junction conditions (A4) and equations on the brane (8), we can deduce the following equations:

$$
\begin{aligned}
& \ddot{\alpha}_{0}=\frac{\kappa^{2} \alpha_{1}}{2}(\rho+p)=\alpha_{1}\left(\beta_{1}-\alpha_{1}\right), \\
& \alpha_{1}^{2}=\frac{1}{l^{2}}+\dot{\alpha}_{0}^{2}, \\
& \frac{\beta_{1}}{\alpha_{1}}=1+\frac{\ddot{\alpha}_{0}}{\alpha_{1}^{2}} \\
& \dot{\alpha}_{1}=\frac{\dot{\alpha}_{0} \ddot{\alpha}_{0}}{\alpha_{1}}=\dot{\alpha}_{0}\left(\beta_{1}-\alpha_{1}\right) .
\end{aligned}
$$

To calculate the perturbations, we need $\alpha_{2}$ and $\beta_{2}$. From the $y^{0}$ th order of the $(0,0)$ and $(i, j)$ components of the Einstein equations, we can write $\alpha_{2}$ and $\beta_{2}$ in terms of $\alpha_{0}, \alpha_{1}$, and $\beta_{1}$ :

$$
\begin{aligned}
& \alpha_{2}=\dot{\alpha}_{0}^{2}-2 \alpha_{1}^{2}+\alpha_{1} \beta_{1}+\frac{2}{l^{2}}, \\
& \beta_{2}=\dot{\alpha}_{0}^{2}+2 \ddot{\alpha}_{0}+\alpha_{1}^{2}-2 \alpha_{1} \beta_{1}+\frac{2}{l^{2}} .
\end{aligned}
$$

\section{APPENDIX B: DERIVATION OF THE EFFECTIVE EINSTEIN EQUATIONS (40) AND (41)}

In this Appendix we review the formalism used to solve the perturbations in the bulk and impose the junction conditions developed in [11]. Using the formalism, we obtain the effective Einstein equations (40) and (41).

\section{Review of the formalism}

First let us review the formalism for obtaining the perturbations in the bulk. We start with the perturbed AdS spacetime in Poincaré coordinates:

$$
\begin{aligned}
d s^{2}= & \left(\begin{array}{l}
l \\
z
\end{array}\right)^{2}\left\{d z^{2}-(1+2 \phi) d \tau^{2}+2 b_{, i} d x^{i} d \tau\right. \\
& \left.+\left[(1-2 \hat{\Psi}) \delta_{i j}+2 \hat{E}_{, i j}\right] d x^{i} d x^{j}\right\} .
\end{aligned}
$$

Here $\phi, b, \hat{\Psi}$, and $\hat{E}$ are given by

$$
\begin{gathered}
h=\left(\frac{z}{l}\right)^{2} \int \frac{d^{3} \mathbf{k}}{(2 \pi)^{3}} \int d m h(m, \mathbf{k}) Z_{2}(m z) e^{-i \omega \tau} e^{i \mathbf{k} \cdot \mathbf{x}} \\
(h=\phi, b, \hat{\Psi}, \hat{E}),
\end{gathered}
$$


where $Z_{2}$ is a combination of the Hankel functions of the first and second kinds of the second rank, $Z_{2}(m z)$ $=H_{2}^{(1)}(m z)+a(m) H_{2}^{(2)}(m z)$. Here we used the transversetraceless gauge conditions

$$
\begin{array}{r}
\phi-3 \hat{\Psi}+\nabla^{2} \hat{E}=0, \\
2 \frac{d \phi}{d \tau}+\nabla^{2} b=0, \\
\frac{d b}{d \tau}+2 \hat{\Psi}-2 \nabla^{2} \hat{E}=0 .
\end{array}
$$

Thus the coefficients $h(m, \mathbf{k})$ satisfy

$$
\begin{aligned}
\phi(m, \mathbf{k}) & =\frac{2 \mathbf{k}^{4}}{3 m^{2}} l^{2} E(m, \mathbf{k}), \\
b(m, \mathbf{k}) & =-4 i \frac{\sqrt{\mathbf{k}^{2}+m^{2}} \mathbf{k}^{2} l^{2}}{3 m^{2}} E(m, \mathbf{k}), \\
\hat{\Psi}(m, \mathbf{k}) & =-\frac{\mathbf{k}^{2} l^{2}}{3} E(m, \mathbf{k}), \\
\hat{E}(m, \mathbf{k}) & =\frac{2 \mathbf{k}^{2}+3 m^{2}}{3 m^{2}} l^{2} E(m, \mathbf{k}),
\end{aligned}
$$

where $E(m, \mathbf{k})$ is an arbitrary coefficient.

The perturbation in the metric (4) is obtained by the coordinate transformation

$$
z=z(y, t)=l e^{-\alpha(y, t)}, \quad \tau=\tau(y, t) .
$$

The Jacobian of the transformation is given by

$$
\begin{aligned}
& \frac{\partial \tau}{\partial y}=l \dot{\alpha} e^{-\alpha}, \quad \frac{\partial z}{\partial y}=-l \alpha^{\prime} e^{-\alpha}, \\
& \frac{\partial \tau}{\partial t}=l \alpha^{\prime} e^{-\alpha}, \quad \frac{\partial z}{\partial t}=-l \dot{\alpha} e^{-\alpha} .
\end{aligned}
$$

Note that at late times

$$
\frac{d T}{d t}=l \alpha_{1} e^{-\alpha_{0}}=-e^{-\alpha_{0}}
$$

Thus we obtain $T=\tau(0, t)=-\eta$, where $\eta$ is the conformal time. After the coordinate transformation, the resulting metric is given by

$$
\begin{aligned}
d s^{2}= & e^{2 \beta(y, t)}\left[(1+2 \hat{N}) d y^{2}-(1+2 \hat{\Phi}) d t^{2}+2 \hat{A} d t d y\right] \\
& +e^{2 \alpha(y, t)}\left\{\left[(1-2 \hat{\Psi}) \delta_{i j}+2 \hat{E}_{, i j}\right] d x^{i} d x^{j}+2 \hat{B}_{, i} d x^{i} d t\right. \\
& \left.+2 \hat{G}_{, i} d x^{i} d y\right\},
\end{aligned}
$$

$$
\begin{aligned}
& \hat{\Phi}=\left(l \alpha^{\prime}\right)^{2} e^{-2 \beta} \phi, \\
& \hat{B}=\left(l \alpha^{\prime}\right) e^{-\alpha} b, \\
& \hat{N}=-(l \dot{\alpha})^{2} e^{-2 \beta} \phi, \\
& \hat{A}=-2\left(l^{2} \dot{\alpha} \alpha^{\prime}\right) e^{-2 \beta} \phi, \\
& \hat{G}=(l \dot{\alpha}) e^{-\alpha} b .
\end{aligned}
$$

There are three degrees of freedom in the gauge transformations:

$$
x^{M} \rightarrow x^{M}+\xi^{M}, \quad \xi^{M}=\left(\xi^{y}, \xi^{t}, \xi^{, i}\right) .
$$

After this gauge transformation, the perturbed metric is given by

$$
\begin{aligned}
d s^{2}= & e^{2 \beta(y, t)}\left[(1+2 N) d y^{2}-(1+2 \Phi) d t^{2}+2 A d t d y\right] \\
& +e^{2 \alpha(y, t)}\left\{\left[(1-2 \Psi) \delta_{i j}+2 E_{, i j}\right] d x^{i} d x^{j}+2 B_{, i} d x^{i} d t\right. \\
& \left.+2 G_{, i} d x^{i} d y\right\}
\end{aligned}
$$

where

$$
\begin{aligned}
& \Phi=\hat{\Phi}+\dot{\xi}^{t}+\beta^{\prime} \xi^{y}+\dot{\beta} \xi^{t}, \\
& \Psi=\hat{\Psi}-\dot{\alpha} \xi^{t}-\alpha^{\prime} \xi^{y}, \\
& E=\hat{E}+\xi, \\
& B=\hat{B}+\dot{\xi}-e^{2(\beta-\alpha)} \xi^{t}, \\
& A=\hat{A}+\dot{\xi}^{y}-\xi^{t^{\prime}}, \\
& G=\hat{G}+e^{2(\beta-\alpha)} \xi^{y}+\xi^{\prime}, \\
& N=\hat{N}+\xi^{y^{\prime}}+\dot{\beta} \xi^{t}+\beta^{\prime} \xi^{y} .
\end{aligned}
$$

Using these degrees of freedom, we perform the gauge transformation to the Gaussian normal (GN) coordinates in which the junction conditions will be imposed. In the GN coordinates, the transverse components of the metric vanish ( $G$ $=A=0$ ) and the brane is located at $y=0$. The former conditions are achieved by $\xi$ and $\xi^{t}$. We can also take the gauge $E_{0}=B_{0}=0$ using the residual gauge transformations in $\xi$ and $\xi^{t}$. Then $\xi$ and $\xi^{t}$ are determined in terms of $\xi^{y}$ as

$$
\begin{aligned}
\xi^{t} & =\int_{0}^{y} d y\left(\hat{A}+\dot{\xi}^{y}\right)+\hat{T}_{0}, \quad \hat{T}_{0}=e^{2 \alpha_{0}}\left(\hat{B}_{0}-\hat{E}_{0}\right), \\
\xi & =-\int_{0}^{y} d y\left(\hat{G}+e^{2(\beta-\alpha)} \xi^{y}\right)-\hat{E}_{0} .
\end{aligned}
$$

The condition that the brane is located at $y=0$ is achieved by $\xi^{y}$ which will be determined by the matter perturbations on the brane.

The metric perturbations on the brane are obtained as

where 


$$
\begin{aligned}
& \Phi_{0}=\hat{\Phi}_{0}+\beta_{1} \xi_{0}^{y}+\hat{T}_{0}, \\
& \Psi_{0}=\hat{\Psi}_{0}-\alpha_{1} \xi_{0}^{y}-\dot{\alpha}_{0} \hat{T}_{0}, \\
& N_{0}=\hat{N}_{0}+\xi_{1}^{y}+\beta_{1} \xi_{0}^{y},
\end{aligned}
$$

and the first derivatives of the metric perturbations are given by

$$
\begin{aligned}
\Phi_{1}= & \ddot{\xi}_{0}^{y}+\beta_{1} \xi_{1}^{y}+\beta_{2} \xi_{0}^{y}+\hat{\Phi}_{1}+\dot{\hat{A}}_{0}+\dot{\beta}_{1} \hat{T}_{0}, \\
\Psi_{1}= & -\alpha_{1} \xi_{1}^{y}-\dot{\alpha}_{0} \xi_{0}^{y}-\alpha_{2} \xi_{0}^{y}+\hat{\Psi}_{1}-\dot{\alpha}_{0} \hat{A}_{0}-\dot{\alpha}_{1} \hat{T}_{0}, \\
N_{1}= & \xi_{2}^{y}+\beta_{1} \xi_{1}^{y}+\beta_{2} \xi_{0}^{y}+\hat{N}_{1}+\dot{\beta}_{1} \hat{T}_{0}, \\
B_{1}= & e^{-2 \alpha_{0}}\left(-2 \xi_{0}^{y}+2 \dot{\alpha}_{0} \xi_{0}^{y}-2\left(\beta_{1}-\alpha_{1}\right) \hat{T}_{0}-\hat{A}_{0}\right. \\
& \left.+e^{2 \alpha_{0}} \hat{B}_{1}-e^{2 \alpha_{0}} \hat{G}_{0}\right), \\
E_{1}= & \hat{E}_{1}-e^{-2 \alpha_{0}} \xi_{0}^{y}-\hat{G}_{0} .
\end{aligned}
$$

Combining Eq. (B15) with the junction conditions [19]

$$
\begin{aligned}
& \Psi_{1}=-\alpha_{1} N_{0}+\frac{1}{6} \kappa^{2} \delta \rho, \\
& \Phi_{1}=\beta_{1} N_{0}+\kappa^{2}\left(\frac{\delta \rho}{3}+\frac{\delta p}{2}\right), \\
& B_{1}=-2\left(\beta_{1}-\alpha_{1}\right) e^{-\alpha_{0}} v, \\
& E_{1}=0,
\end{aligned}
$$

we can write the matter perturbations in terms of $\xi_{0}^{y}$ and $E(m, \mathbf{k})$ :

$$
\begin{aligned}
\kappa^{2} \delta \rho= & -6\left[\dot{\alpha}_{0} \dot{\xi}_{0}^{y}+\left(\alpha_{2}-\alpha_{1} \beta_{1}\right) \xi_{0}^{y}-\hat{\Psi}_{1}+\dot{\alpha}_{0} \hat{A}_{0}+\dot{\alpha}_{1} \hat{T}_{0}\right. \\
& \left.-\alpha_{1} \hat{N}_{0}\right], \\
\kappa^{2} \delta p= & 2\left[\ddot{\xi}_{0}^{y}+2 \dot{\alpha}_{0} \dot{\xi}_{0}^{y}+\left(2 \alpha_{2}+\beta_{2}-\beta_{1}^{2}-2 \alpha_{1} \beta_{1}\right) \xi_{0}^{y}+\hat{\Phi}_{1}\right. \\
& -2 \hat{\Psi}_{1}+\hat{A}_{0}+2 \dot{\alpha}_{0} \hat{A}_{0}+\left(\dot{\beta}_{1}+2 \dot{\alpha}_{1}\right) \hat{T}_{0} \\
& \left.-\left(\beta_{1}+2 \alpha_{1}\right) \hat{N}_{0}\right],
\end{aligned}
$$

$\kappa^{2}(\rho+p) e^{\alpha_{0}} v$

$$
\begin{aligned}
= & 2 \dot{\xi}_{0}^{y}-2 \dot{\alpha}_{0} \xi_{0}^{y}-e^{2 \alpha_{0}} \hat{B}_{1}+2\left(\beta_{1}-\alpha_{1}\right) \hat{T}_{0}+e^{2 \alpha_{0}} \hat{G}_{0} \\
& +\hat{A}_{0}, \\
0= & -2 e^{-2 \alpha_{0}} \xi_{0}^{y}+2 \hat{E}_{1}-2 \hat{G}_{0},
\end{aligned}
$$

where $\hat{T}_{0}=e^{2 \alpha_{0}}\left(\hat{B}_{0}-\hat{E}_{0}\right)$. From the last equation in (B17), $\xi_{0}^{y}$ is written using $E(m, \mathbf{k})$. Thus the matter perturbations are written in terms of the perturbations in the bulk (B2) and
(B4). These equations correspond to Eq. (14) in the background spacetime. The solutions for the perturbations are obtained by determining $E(m, \mathbf{k})$ and $a(m)$ by imposing the equations of state of the matter perturbations such as $\delta p$ $=c_{s}^{2} \delta \rho$ and the appropriate boundary conditions in the bulk. In [11], $E(m, \mathbf{k})$ is obtained for perturbations at superhorizon scales in the low energy universe with a constant barotropic parameter. The boundary condition was taken so that the perturbations are outgoing at the horizon of the AdS spacetime. In general, however, it is rather difficult to obtain the solution for $E(m, \mathbf{k})$. Thus we use the method described in Sec. II. We rewrite Eq. (B17) as the effective Einstein equations. To do so, we should rewrite the right-hand side of Eq. (B17) in terms of the metric perturbations $\Phi_{0}$ and $\Psi_{0}$.

\section{Derivation of the equations (40) and (41)}

We rewrite the right-hand side of Eqs. (B17) by using metric perturbations $\Phi_{0}$ and $\Psi_{0}$ to derive Eqs. (40) and (41). We will write $\hat{\Phi}, \hat{B}, \hat{N}, \hat{A}$, and $\hat{G}$ in terms of $\phi$ and $b$ using Eq. (B9). First let us consider the density perturbation $\delta \rho$. From Eq. (B17), $\delta \rho$ is given by

$$
\begin{aligned}
\kappa^{2} \delta \rho= & -6\left(\dot{\alpha}_{0} \dot{\xi}_{0}^{y}-\dot{\alpha}_{0}^{2} \xi_{0}^{y}-\dot{\alpha}_{0}^{2} \alpha_{1} l^{2} \phi_{0}+\dot{\alpha}_{0} \ddot{\alpha}_{0} e^{\alpha_{0}} l b_{0}\right. \\
& \left.-\frac{\dot{\alpha}_{0} \ddot{\alpha}_{0}}{\alpha_{1}} e^{2 \alpha_{0}} \hat{E}_{0}-\hat{\Psi}_{1}\right),
\end{aligned}
$$

where we used Eq. (A13) to write $\alpha_{2}-\alpha_{1} \beta_{1}=-\dot{\alpha}_{0}{ }^{2}$ and $\dot{\alpha}_{1}=\dot{\alpha}_{0} \ddot{\alpha}_{0} / \alpha_{1}$. The strategy is to write $\xi_{0}^{y}$ using $\Psi_{0}$ and $\Phi_{0}$. From Eq. (B14), the metric perturbations $\Psi_{0}$ and $\Phi_{0}$ are given by

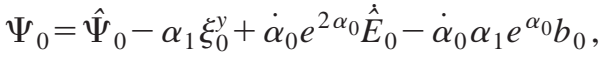

$$
\begin{aligned}
& \Phi_{0}=\alpha_{1}^{2} l^{2} \phi_{0}+\left(1+\frac{\ddot{\alpha}_{0}}{\alpha_{1}^{2}}\right) \alpha_{1} \xi_{0}^{y}-e^{2 \alpha_{0}} \ddot{\hat{E}}_{0} \\
& -2 \dot{\alpha}_{0} e^{2 \alpha_{0}} \dot{\hat{E}}_{0}+\left(\alpha_{1} \dot{\alpha}_{0}+\dot{\alpha}_{1}\right) l e^{\alpha_{0}} b_{0} \\
& +\alpha_{1} e^{\alpha_{0}} l \dot{b}_{0},
\end{aligned}
$$

where we used Eq. (A12) to write $\beta_{1}=\left(1+\ddot{\alpha}_{0} / \alpha_{1}^{2}\right) \alpha_{1}$. From Eq. (B19), we can show that

$$
\begin{aligned}
\dot{\alpha}_{0} \dot{\Psi}_{0}+\dot{\alpha}_{0}^{2} \Phi_{0}= & -\alpha_{1}\left(\dot{\alpha}_{0} \dot{\xi}_{0}^{y}-\dot{\alpha}_{0}^{2} \xi_{0}^{y}-\dot{\alpha}_{0}^{2} \alpha_{1} l^{2} \phi_{0}\right. \\
& \left.+\dot{\alpha}_{0} \ddot{\alpha}_{0} e^{\alpha_{0}} l b_{0}-\frac{\dot{\alpha}_{0} \ddot{\alpha}_{0}}{\alpha_{1}} e^{2 \alpha_{0}} \dot{\hat{E}}_{0}\right)+\dot{\alpha}_{0} \dot{\hat{\Psi}}_{0}
\end{aligned}
$$

Thus the terms written using $\xi_{0}^{y}$ in Eq. (B18) can be rewritten using $\Phi_{0}$ and $\Psi_{0}$. We obtain 


$$
-\frac{\alpha_{1} \kappa^{2}}{2} \delta \rho=-3\left(\dot{\alpha}_{0} \dot{\Psi}_{0}+\dot{\alpha}_{0}^{2} \Phi_{0}+\alpha_{1} \hat{\Psi}_{1}-\dot{\alpha}_{0} \dot{\hat{\Psi}}_{0}\right)
$$

The remaining task is to rewrite the terms written in terms of $\hat{\Psi}$ in terms of $\Psi_{0}$. First, using the solution of the perturbations (B2) and (B4), we rewrite $\Psi_{0}$ in terms of $E(m, \mathbf{k})$. From Eq. (B17), $\xi_{0}^{y}$ is given by

$$
\xi_{0}^{y}=e^{2 \alpha_{0}} \hat{E}_{1}-\dot{\alpha}_{0} e^{\alpha_{0}} l b_{0} .
$$

Then we can rewrite $\Psi_{0}$ as

$$
\Psi_{0}=\hat{\Psi}_{0}-\alpha_{1} e^{2 \alpha_{0}} \hat{E}_{1}+\dot{\alpha}_{0} e^{2 \alpha_{0}} \dot{\hat{E}}_{0} .
$$

Using the Jacobian of the transformation (B6) and

$$
\frac{d}{d z}\left[z^{2} Z_{2}(m z)\right]=m z^{2} Z_{1}(m z)
$$

we can get the following equations:

$$
\begin{aligned}
\alpha_{1} e^{2 \alpha_{0}} \hat{E}_{1}(\mathbf{k})= & -\int d m \hat{E}(m, \mathbf{k})\left[\alpha_{1}^{2} m l e^{-\alpha_{0}} Z_{1}\left(m l e^{-\alpha_{0}}\right)\right. \\
& \left.+\dot{\alpha}_{0} \alpha_{1} i \omega l e^{-\alpha_{0}} Z_{2}\left(m l e^{-\alpha_{0}}\right)\right] e^{-i \omega T}, \\
\dot{\alpha}_{0} e^{2 \alpha_{0}} \hat{\hat{E}}_{0}(\mathbf{k})= & -\int d m \hat{E}(m, \mathbf{k})\left[\dot{\alpha}_{0}{ }^{2} m l e^{-\alpha_{0}} Z_{1}\left(m l e^{-\alpha_{0}}\right)\right. \\
& \left.+\dot{\alpha}_{0} \alpha_{1} i \omega l e^{-\alpha_{0}} Z_{2}\left(m l e^{-\alpha_{0}}\right)\right] e^{-i \omega T} .(\mathrm{B} 25
\end{aligned}
$$

Thus we find

$$
\begin{aligned}
-\alpha_{1} e^{2 \alpha_{0}} \hat{E}_{1}+\dot{\alpha}_{0} e^{2 \alpha_{0} \hat{E}_{0}}= & \int d m E(m, \mathbf{k})\left(\frac{2 \mathbf{k}^{2}+3 m^{2}}{3 m} l e^{-\alpha_{0}}\right) \\
& \times Z_{1}\left(m l e^{-\alpha_{0}}\right) e^{-i \omega T}, \quad(\mathrm{~B} 26)
\end{aligned}
$$

where we used Eq. (B4) and $\alpha_{1}^{2}=1 / l^{2}+\dot{\alpha}_{0}^{2}$ [Eq. (A12)]. $\hat{\Psi}_{0}$ is given by

$$
\begin{aligned}
& \hat{\Psi}_{0}(\mathbf{k})=e^{-2 \alpha_{0}} \int d m \hat{\Psi}_{0}(m, \mathbf{k}) Z_{2}\left(m l e^{-\alpha_{0}}\right) e^{-i \omega T} \\
& =-\int d m E(m, \mathbf{k})\left(\frac{2 \mathbf{k}^{2}}{3 m} l e^{-\alpha_{0}} Z_{1}\left(m l e^{-\alpha_{0}}\right)\right. \\
& \left.-\frac{1}{3}\left(\mathbf{k} l e^{-\alpha_{0}}\right)^{2} Z_{0}\left(m l e^{-\alpha_{0}}\right)\right) e^{-i \omega T},
\end{aligned}
$$

where we used Eq. (B4) and $Z_{2}(m z)=(2 / m z) Z_{1}(m z)$ $-Z_{0}(m z)$. Then we can write $\Psi_{0}$ in terms of $E(m, \mathbf{k})$ as

$$
\begin{aligned}
\Psi_{0}(\mathbf{k})= & \int d m E(m, \mathbf{k})\left(m l e^{-\alpha_{0}} Z_{1}\left(m l e^{-\alpha_{0}}\right)\right. \\
& \left.+\frac{1}{3}\left(\mathbf{k} l e^{-\alpha_{0}}\right)^{2} Z_{0}\left(m l e^{-\alpha_{0}}\right)\right) e^{-i \omega T(t)} .
\end{aligned}
$$

On the other hand, the same calculation as for Eq. (B26) yields

$$
\begin{aligned}
\alpha_{1} \hat{\Psi}_{1}(\mathbf{k})-\dot{\alpha}_{0} \dot{\hat{\Psi}}_{0}(\mathbf{k})= & \frac{1}{3} \int d m E(m, \mathbf{k}) \mathbf{k}^{2} m l e^{-3 \alpha_{0}} \\
& \times Z_{1}\left(m l e^{-\alpha_{0}}\right) e^{-i \omega T(t)}
\end{aligned}
$$

Thus Eq. (B21) becomes

$$
\begin{aligned}
-\frac{\kappa^{2} \alpha_{1}}{2} \delta \rho(\mathbf{k})= & -3\left(\dot{\alpha}_{0} \dot{\Psi}_{0}+\dot{\alpha}_{0}^{2} \Phi_{0}\right)-e^{-2 \alpha_{0}} \mathbf{k}^{2} \Psi_{0} \\
& +\frac{1}{3} e^{-4 \alpha_{0}} \int d m E(m, \mathbf{k}) \mathbf{k}^{4} l^{2} Z_{0}\left(m l e^{-\alpha_{0}}\right) \\
& \times e^{-i \omega T(t)}
\end{aligned}
$$

The other quantities $\delta p$ and $v$ can be calculated in the same way. The calculations are straightforward but lengthy. It is easier to derive $\delta p$ and $v$ using the equations on the brane (19), (20), and (21). The pressure perturbations $\delta p$ can be obtained from Eq. (19) as

$$
\begin{aligned}
-\frac{\kappa^{2} \alpha_{1}}{2} \delta p(\mathbf{k})= & \ddot{\Psi}_{0}+4 \dot{\alpha}_{0} \dot{\Psi}_{0}+\dot{\alpha}_{0} \dot{\Phi}_{0}+2\left(\ddot{\alpha}_{0}+2 \dot{\alpha}_{0}^{2}\right) \Phi_{0} \\
& +\frac{1}{3} e^{-2 \alpha_{0}}\left(2 \mathbf{k}^{2} \Psi_{0}-\mathbf{k}^{2} \Phi_{0}\right)-\frac{\kappa^{2} \beta_{1}}{6} \delta \rho(\mathbf{k}) .
\end{aligned}
$$

Substituting Eq. (B30) into Eq. (B31), we get $\delta p$ as

$$
\begin{aligned}
-\frac{\kappa^{2} \alpha_{1}}{2} \delta p(\mathbf{k})= & \dot{\Psi}_{0}+\left(3 \dot{\alpha}_{0}-\frac{\dot{\alpha}_{0} \ddot{\alpha}_{0}}{\alpha_{1}^{2}}\right) \dot{\Psi}_{0}+\dot{\alpha}_{0} \dot{\Phi}_{0} \\
& +\left(2 \ddot{\alpha}_{0}-\frac{\dot{\alpha}_{0}^{2} \ddot{\alpha}_{0}}{\alpha_{1}^{2}}+3 \dot{\alpha}_{0}^{2}\right) \Phi_{0}-\frac{1}{3} e^{-2 \alpha_{0}} \mathbf{k}^{2} \Phi_{0} \\
& +\frac{1}{3}\left(1-\frac{\ddot{\alpha}_{0}}{\alpha_{1}^{2}}\right) e^{-2 \alpha_{0}} \mathbf{k}^{2} \Psi_{0} \\
& +\frac{1}{9}\left(1+\frac{\ddot{\alpha}_{0}}{\alpha_{1}^{2}}\right) e^{-4 \alpha_{0}} \\
& \times \int d m E(m, \mathbf{k}) \mathbf{k}^{4} l^{2} Z_{0}\left(m l e^{\left.-\alpha_{0}\right)}\right. \\
& \times e^{-i \omega T(t)} .
\end{aligned}
$$

The velocity perturbation $v$ is also obtained from the equation on the brane (21):

$$
\begin{aligned}
(\rho+p) e^{\alpha_{0}} v= & \mathbf{k}^{-2} e^{2 \alpha_{0}}\left[-\dot{\delta} \rho+3(\rho+p) \dot{\Psi}_{0}\right. \\
& \left.-3 \dot{\alpha}_{0}(\delta \rho+\delta p)\right] .
\end{aligned}
$$

Substituting Eqs. (B30) and (B32) into Eq. (B33), we can show that $v$ is given by 


$$
-\frac{\kappa^{2} \alpha_{1}}{2}(\rho+p) e^{\alpha_{0}} v(\mathbf{k})=\dot{\Psi}_{0}+\dot{\alpha}_{0} \Phi_{0}+\Delta v
$$

where $\Delta v$ is given by

$$
\begin{aligned}
\Delta v= & -\mathbf{k}^{-2} e^{-2 \alpha_{0}} \frac{d}{d t}\left[\frac{1}{3} \int d m E(m, \mathbf{k}) \mathbf{k}^{4} l^{2} Z_{0}\left(m l e^{-\alpha_{0}}\right)\right. \\
& \left.\times e^{-i \omega T(t)}\right] \\
= & \frac{1}{3} e^{-3 \alpha_{0}} \int d m E(m, \mathbf{k})\left[\alpha_{1} i \omega \mathbf{k}^{2} l^{3} Z_{0}\left(m l e^{-\alpha_{0}}\right)\right. \\
& \left.-\dot{\alpha}_{0} m \mathbf{k}^{2} l^{3} Z_{1}\left(m l e^{-\alpha_{0}}\right)\right] e^{-i \omega T(t)} .
\end{aligned}
$$

Finally, let us rewrite the metric perturbations $\Phi_{0}$ in terms of $E(m, \mathbf{k})$ to derive Eq. (41). From Eqs. (B19) and (B22), $\Phi_{0}$ is given by

$$
\begin{aligned}
\Phi_{0}= & \alpha_{1}^{2} l^{2} \phi_{0}+\alpha_{1} e^{\alpha_{0}} l \dot{b}_{0}+\left(1+\frac{\ddot{\alpha}_{0}}{\alpha_{1}^{2}}\right) \alpha_{1} e^{2 \alpha_{0}} \hat{E}_{1}-2 \dot{\alpha}_{0} e^{2 \alpha_{0}} \hat{\hat{E}}_{0} \\
& -e^{2 \alpha_{0} \ddot{E}_{0}}
\end{aligned}
$$

From Eq. (B25),

$$
\begin{aligned}
(1+ & \left.\frac{\ddot{\alpha}_{0}}{\alpha_{1}^{2}}\right) \alpha_{1} e^{2 \alpha_{0}} \hat{E}_{1}-2 \dot{\alpha}_{0} e^{2 \alpha_{0}} \hat{\hat{E}}_{0}-e^{2 \alpha_{0} \ddot{E}_{0}} \\
= & \int d m E(m, \mathbf{k})\left(\left(\alpha_{1} l\right)^{2} \frac{2 \mathbf{k}^{2}+m^{2}}{m} l e^{-\alpha_{0}} Z_{1}\left(m l e^{-\alpha_{0}}\right)\right. \\
& -\left(\omega^{2} \alpha_{1}^{2}+m^{2} \dot{\alpha}_{0}^{2}\right) l^{4} e^{-2 \alpha_{0}} Z_{0}\left(m l e^{-\alpha_{0}}\right) \\
& \left.-2\left(\alpha_{1} \dot{\alpha}_{0} l^{2}\right) i \omega m l^{2} e^{-2 \alpha_{0}} Z_{1}\left(m l e^{-\alpha_{0}}\right)\right) \\
& \times \frac{2 \mathbf{k}^{2}+3 m^{2}}{3 m^{2}} e^{-i \omega T},
\end{aligned}
$$

where we used $d\left[z Z_{1}(m z)\right] / d z=m z Z_{0}(m z)$. In addition, we can show that

$$
\begin{aligned}
\alpha_{1}^{2} l^{2} \phi_{0}= & \left(\alpha_{1} l\right)^{2} \int d m E(m, \mathbf{k})\left(\frac{4 \mathbf{k}^{4}}{3 m^{3}} l e^{-\alpha_{0}} Z_{1}\left(m l e^{-\alpha_{0}}\right)\right. \\
& \left.-\frac{2 \mathbf{k}^{4}}{3 m^{2}} l^{2} e^{-2 \alpha_{0}} Z_{0}\left(m l e^{-\alpha_{0}}\right)\right) e^{-i \omega T},
\end{aligned}
$$

$$
\begin{aligned}
\alpha_{1} e^{\alpha_{0}} l \dot{b}_{0}= & \int d m E(m, \mathbf{k})\left(-\left(\alpha_{1} l\right)^{2} \frac{8 \omega^{2} \mathbf{k}^{2}}{3 m^{3}} l e^{-\alpha_{0}}\right. \\
& \times Z_{1}\left(m l e^{-\alpha_{0}}\right)+\left(\alpha_{1} l\right)^{2} \frac{4 \omega^{2} \mathbf{k}^{2}}{3 m^{2}} l^{2} e^{-2 \alpha_{0}} \\
& \times Z_{0}\left(m l e^{-\alpha_{0}}\right)+\left(\alpha_{1} \dot{\alpha}_{0} l^{2}\right) \frac{4 i \omega \mathbf{k}^{2}}{3 m} l^{2} e^{-2 \alpha_{0}} \\
& \left.\times Z_{1}\left(m l e^{-\alpha_{0}}\right)\right) e^{-i \omega T},
\end{aligned}
$$

where we used $Z_{2}(m z)=(2 / m z) Z_{1}(m z)-Z_{0}(m z)$. Then $\Phi_{0}$ can be written using $E(m, \mathbf{k})$ as

$$
\begin{aligned}
\Phi_{0}(\mathbf{k})= & \int d m E(m, \mathbf{k})\left(m l e^{-\alpha_{0}} Z_{1}\left(m l e^{-\alpha_{0}}\right)\right. \\
& \left.-\frac{1}{3}\left(\mathbf{k}^{2}+3 m^{2}\right) l^{2} e^{-2 \alpha_{0}} Z_{0}\left(m l e^{-\alpha_{0}}\right)\right) e^{-i \omega T(t)} \\
& +\left(\dot{\alpha}_{0} l\right)^{2} \int d m E(m, \mathbf{k})\left[m l e^{-\alpha_{0}} Z_{1}\left(m l e^{-\alpha_{0}}\right)\right. \\
& \left.-\left(\mathbf{k}^{2}+2 m^{2}\right) l^{2} e^{-2 \alpha_{0}} Z_{0}\left(m l e^{-\alpha_{0}}\right)\right] e^{-i \omega T(t)} \\
& -2 \alpha_{1} \dot{\alpha}_{0} l^{2} \int d m E(m, \mathbf{k}) \\
& \times\left(i \omega m l^{2} e^{-2 \alpha_{0}}\right) Z_{1}\left(m l e^{-\alpha_{0}}\right) e^{-i \omega T(t)}
\end{aligned}
$$

where we used $\left(\alpha_{1} l\right)^{2}=1+\left(\dot{\alpha}_{0} l\right)^{2}$.

\section{APPENDIX C: PRIMORDIAL FLUCTUATIONS}

The CMB anisotropies at large scales are determined by $\zeta_{*}$ which should be determined by the primordial fluctuations. We consider the inflaton $\phi$ confined to the brane with potential $V(\phi)$ [38]. The background equations are given by

$$
\begin{aligned}
\ddot{\phi}+3 \dot{\alpha}_{0} \dot{\phi} & =-\frac{d V(\phi)}{d \phi}, \\
\frac{\kappa^{2} \alpha_{1}}{2} \dot{\phi}^{2} & =\ddot{\alpha}_{0} .
\end{aligned}
$$

The perturbed energy-momentum tensor of the inflaton is given by

$$
\begin{aligned}
\delta \rho & =-\dot{\phi}^{2} \Phi_{0}+\dot{\phi} \dot{\delta} \phi+V^{\prime}(\phi) \delta \phi, \\
\delta p & =-\dot{\phi}^{2} \Phi_{0}+\dot{\phi} \delta \bar{\phi}-V^{\prime}(\phi) \delta \phi, \\
(\rho+p) e^{\alpha_{0}} v & =\dot{\phi} \delta \phi,
\end{aligned}
$$

where $\delta \phi$ are the fluctuations of the inflaton. It is useful to use the Mukhanov variable to describe the evolution of the perturbations: 


$$
Q=\delta \phi+\frac{\dot{\phi}}{\dot{\alpha}_{0}} \Psi_{0}
$$

Combining Eqs. (C2) and (40) and the equation of motion for $\delta \phi$,

$$
\begin{gathered}
\ddot{\delta} \phi+3 \dot{\alpha}_{0} \dot{\delta} \phi+e^{-2 \alpha_{0}} \mathbf{k}^{2} \delta \phi+V^{\prime \prime}(\phi) \delta \phi \\
=3 \dot{\phi} \dot{\Psi}_{0}+\dot{\phi} \Phi_{0}-2 V^{\prime}(\phi) \Phi_{0},
\end{gathered}
$$

we can obtain the evolution equation for $Q$ as

$$
\begin{aligned}
\ddot{Q}+3 & \dot{\alpha}_{0} \dot{Q}+e^{-2 \alpha_{0}} \mathbf{k}^{2} Q+\left(\frac{\dot{\alpha}_{0}}{\dot{\alpha}_{0}}-2 \frac{\ddot{\alpha}_{0}}{\dot{\alpha}_{0}} \frac{V^{\prime}(\phi)}{\dot{\phi}}-2\left(\frac{\ddot{\alpha}_{0}}{\dot{\alpha}_{0}}\right)^{2}\right. \\
& \left.+V^{\prime \prime}(\phi)\right) Q=J
\end{aligned}
$$

where

$$
\begin{aligned}
J= & \frac{\dot{\phi}_{0}}{\dot{\alpha}_{0}}\left[\left(\frac{2 \ddot{\alpha}_{0}}{\ddot{\alpha}_{0}}-\frac{\ddot{\alpha}_{0}}{\dot{\alpha}_{0}}\right) \Delta v+\frac{1}{3} e^{-2 \alpha_{0}} \mathbf{k}^{2} \Phi_{0}-\frac{1}{3}\left(1-\frac{\ddot{\alpha}_{0}}{\alpha_{1}^{2}}\right)\right. \\
& \times e^{-2 \alpha_{0}} \mathbf{k}^{2} \Psi_{0}+\frac{1}{3}\left(\frac{2}{3}-\frac{1}{3} \frac{\ddot{\alpha}_{0}}{\alpha_{1}^{2}}\right) e^{-4 \alpha_{0}} \\
& \left.\times \int d m E(m, \mathbf{k}) \mathbf{k}^{4} l^{2} Z_{0}\left(m l e^{-\alpha_{0}}\right) e^{-i \omega T(t)}\right],
\end{aligned}
$$

and $\Delta v$ is given by Eq. (B35). We take the boundary condition so that $\delta \rho_{\chi}=\delta p_{\chi}=v_{\chi}=0$. At large scales the source term $J$ goes to zero. Then we can find the solution for $Q$ as

$$
Q=\frac{\dot{\phi}}{\dot{\alpha}_{0}}\left(A_{Q}+B_{Q} \int^{t} d t^{\prime} \frac{\dot{\alpha}_{0}^{2}}{e^{3 \alpha_{0}} \dot{\phi}^{2}}\right),
$$

where $A_{Q}$ and $B_{Q}$ are constants of integration. The amplitude $A_{Q}$ of the growing mode solution is determined once $Q$ is quantized. Denoting the power spectrum of $A_{Q}$ as $P_{A_{Q}}$, we get

$$
P_{A_{Q}}=\left.\frac{\dot{\alpha}_{0}}{\dot{\phi}} P_{Q}\right|_{\text {large scales }},
$$

where the right-hand side is the power spectrum of the quantized $Q$ evaluated at large scales. The important point is that $Q$ is related to the Bardeen parameter by Eqs. (C2), (40), and (60) as

$$
Q=\frac{\dot{\phi}}{\dot{\alpha}_{0}}\left(\zeta-\frac{\dot{\alpha}_{0}}{\ddot{\alpha}_{0}} \Delta v\right) \text {. }
$$

Thus at large scales $A_{Q}=\zeta_{*}$ and

$$
P_{\zeta_{*}}=\left.P_{Q}\right|_{\text {large scales }} .
$$

The problem is how to quantize the system of (C5). As in the evolution equation for the density perturbations, the equation becomes nonlocal at scales below $l\left(\mathbf{k} l e^{-\alpha_{0}}>0\right)$. In particular, at high energies, the Hubble horizon is smaller than the curvature scale $l$. Thus even at the horizon scale the corrections are significant. One way is to construct the effective action that gives Eq. (C5) and do path-integral quantization as is done in [28]. Further investigations are needed to obtain the spectrum of $\zeta_{*}$.
[1] V.A. Rubakov and M.E. Shaposhnikov, Phys. Lett. 125B, 136 (1983).

[2] K. Akama, in Gauge Theory and Gravitation, edited by K. Kikkawa, N. Nakanishi, and H. Nariai, Lecture Notes in Physics Vol. 176 (Springer-Verlag, Berlin, 1983), pp. 267-271; hep-th/0001113.

[3] L. Randall and R. Sundrum, Phys. Rev. Lett. 83, 4690 (1999); 83, 3370 (1999).

[4] H.A. Chamblin and H.S. Reall, Nucl. Phys. B562, 133 (1999).

[5] N. Kaloper, Phys. Rev. D 60, 123506 (1999).

[6] T. Nihei, Phys. Lett. B 465, 81 (1999).

[7] H.B. Kim and H.D. Kim, Phys. Rev. D 61, 064003 (2000).

[8] K. Koyama and J. Soda, Phys. Lett. B 483, 432 (2000).

[9] P. Binétruy, C. Deffayet, U. Ellwanger, and D. Langlois, Phys. Lett. B 477, 285 (2000).

[10] E. Flanagan, S. Tye, and I. Wasserman, Phys. Rev. D 62, 024011 (2000).

[11] P. Kraus, J. High Energy Phys. 12, 011 (1999).

[12] D. Vollick, Class. Quantum Grav. 18, 1 (2001).

[13] S. Mukohyama, Phys. Lett. B 473, 241 (2000).
[14] D. Ida, J. High Energy Phys. 09, 014 (2000).

[15] S. Mukohyama, T. Shiromizu, and K. Maeda, Phys. Rev. D 62, 024028 (2000)

[16] J.M. Bardeen, Phys. Rev. D 22, 1882 (1980).

[17] V.F. Mukhanov, H.A. Feldman, and R.H. Brandenberger, Phys. Rep. 215, 203 (1992).

[18] H. Kodama and M. Sasaki, Int. J. Mod. Phys. A 1, 265 (1986).

[19] K. Koyama and J. Soda, Phys. Rev. D 62, 123502 (2000).

[20] S. Mukohyama, Phys. Rev. D 62, 084015 (2000); Class. Quantum Grav. 17, 4777 (2000); Phys. Rev. D 64, 064006 (2001).

[21] H. Kodama, A. Ishibashi, and O. Seto, Phys. Rev. D 62, 064022 (2000); H. Kodama, hep-th/0012132.

[22] C. van de Bruck, M. Dorca, R.H. Brandenberger, and A. Lukas, Phys. Rev. D 62, 123515 (2000); C. van de Bruck and M. Dorca, hep-th/0012073; M. Dorca and C. van de Bruck, Nucl. Phys. B605, 215 (2001).

[23] D. Langlois, Phys. Rev. D 62, 126012 (2000); Phys. Rev. Lett. 86, 2212 (2001).

[24] R. Maartens, Phys. Rev. D 62, 084023 (2000); C. Gordon and R. Maartens, ibid. 63, 044022 (2001). 
[25] N. Deruelle, T. Dolezel, and J. Katz, Phys. Rev. D 63, 083513 (2001).

[26] D. Langlois, R. Maartens, and D. Wands, Phys. Lett. B 489, 259 (2000).

[27] H.A. Bridgman, K.A. Malik, and D. Wands, astro-ph/0107245.

[28] S.W. Hawking, T. Hertog, and H.S. Reall, Phys. Rev. D 62, 043501 (2000).

[29] D. Langlois, R. Maartens, M. Sasaki, and D. Wands, Phys. Rev. D 63, 084009 (2001).

[30] T. Shiromizu, K. Maeda, and M. Sasaki, Phys. Rev. D 62, 024012 (2000).

[31] D.S. Gorbunov, V.A. Rubakov, and S.M. Sibiryakov, hep-th/0108017.

[32] M. Sasaki, T. Shiromizu, and K. Maeda, Phys. Rev. D 62, 024008 (2000).
[33] J. Garriga and T. Tanaka, Phys. Rev. Lett. 84, 2778 (2000).

[34] S. Giddings, E. Katz, and L. Randall, J. High Energy Phys. 03, 023 (2000).

[35] K. Enqvist and H. Kurki-Suonio, Phys. Rev. D 61, 043002 (2000); D. Langlois and A. Riazuelo, ibid. 62, 043504 (2000); L. Amendola, C. Gordon, D. Wands, and M. Sasaki, astro-ph/0107089.

[36] S. Kobayashi, K. Koyama, and J. Soda, Phys. Lett. B 501, 157 (2001).

[37] N. Sago, Y. Himemoto, and M. Sasaki, Phys. Rev. D (to be published) gr-qc/0104033; Y. Himemoto and M. Sasaki, ibid. 63, 044015 (2001).

[38] R. Maartens, D. Wands, B.A. Bassett, and I. Heard, Phys. Rev. D 62, 041301 (2000). 